\title{
Existence, Uniqueness, and Stability Analysis of Impulsive Neural Networks with Mixed Time Delays
}

\author{
Qiang $\mathrm{Xi}^{1,2}$ and Jianguo $\mathrm{Si}^{1}$ \\ ${ }^{1}$ School of Mathematics, Shandong University, Jinan 250100, China \\ ${ }^{2}$ School of Mathematics and Quantitative Economics, Shandong University of Finance and Economics, Jinan 250002, China \\ Correspondence should be addressed to Qiang Xi; xiqiang_2000@163.com
}

Received 22 January 2014; Revised 16 March 2014; Accepted 25 March 2014; Published 15 May 2014

Academic Editor: Ivanka Stamova

Copyright (c) 2014 Q. Xi and J. Si. This is an open access article distributed under the Creative Commons Attribution License, which permits unrestricted use, distribution, and reproduction in any medium, provided the original work is properly cited.

\begin{abstract}
We study a class of impulsive neural networks with mixed time delays and generalized activation functions. The mixed delays include time-varying transmission delay, bounded time-varying distributed delay, and discrete constant delay in the leakage term. By using the contraction mapping theorem, we obtain a sufficient condition to guarantee the global existence and uniqueness of the solution for the addressed neural networks. In addition, a delay-independent sufficient condition for existence of an equilibrium point and some delay-dependent sufficient conditions for stability are derived, respectively, by using topological degree theory and Lyapunov-Krasovskii functional method. The presented results require neither the boundedness, monotonicity, and differentiability of the activation functions nor the differentiability (even differential boundedness) of time-varying delays. Moreover, the proposed stability criteria are given in terms of linear matrix inequalities (LMI), which can be conveniently checked by the MATLAB toolbox. Finally, an example is given to show the effectiveness and less conservativeness of the obtained results.
\end{abstract}

\section{Introduction}

As we know, time delay in a system is a common phenomenon that describes the fact that the future state of the system depends not only on the present state but also on the past state and is always unavoidably encountered in many fields such as automatic control, biological chemistry, physical engineer, and neural networks [1-5]. Moreover, time delays can affect the stability of a neural network and create oscillatory and bad dynamic performance [3-5]. Hence, it is significant and necessary to take into account the delay effects on dynamics of neural networks, for example, existence, uniqueness and stability, and so on. To date, neural network models with two categories of time delays, namely, discrete and continuously distributed time delays, have been extensively investigated by many researchers, using some effective approaches; see [622] and references therein. For instance, in [6], Kharitonov and Zhabko studied the robust stability of time-delay systems via Lyapunov-Krasovskii functional approach. Wu et al. [9] introduced free-weighting matrix approach and investigated the robust stability problem for time-varying delay systems. $\mathrm{Gu}$ introduced the delay decomposition method in [10].
Recently, a special type of time delay, namely, leakage delay (or forgetting delay), is identified and investigated due to its existence in many real systems. In 2007, Gopalsamy [11] proposed the bidirectional associative memory neural networks with constant delay in the leakage term and derived sufficient conditions for existence and stability of equilibrium. Based on this work, Li and Huang [12] investigated the stability of general nonlinear systems with leakage delay, by model transformation, contraction mapping theorem, and degenerate Lyapunov-Krasovskii functional. However, dynamical analysis of neural networks with time delay in leakage term has been little considered due to some theoretical and technical difficulties [23-30]. In fact, time delay in the stabilizing negative feedback term has a tendency to destabilize a system [11] and has great impact on the dynamics of neural networks.

On the other hand, besides delay, impulses are also likely to exist in neural networks. In implementation of electronic networks, the state is subject to instantaneous perturbations and experiences abrupt change at certain moments, which may be caused by switching phenomenon, frequency change, or other sudden noises; that is, it does exhibit impulsive 
effects; see [13-21, 31-35]. Therefore, impulsive perturbations should be taken into account when studying the dynamics of neural networks. Since the existence of delays and impulses is frequently a source of instability, bifurcation, and chaos for dynamical systems, it is significant to study both delay and impulsive effects on dynamical systems [13-21, 25, 26, 32]. In [25], $\mathrm{Li}$ et al. investigated the existence, uniqueness, and stability problems of recurrent neural networks with discrete time delay and time delay in the leakage term under impulsive perturbations, while being without distributed delay. Since neural networks usually have a spatial extent, there is a distribution of propagation delays over a period of time. In these circumstances the signal propagation is not instantaneous and cannot be modelled only with discrete delays and a more appropriate way is to incorporate continuously distributed delays in neural network models. To the best of our knowledge, so far, there has been very little existing work on impulsive neural networks with time delay in the leakage term and discrete and time-varying distributed delays via LMI approach [27].

Motivated by aforementioned discussion, in this paper, we consider a class of impulsive neural networks with mixed time delays and generalized activation functions which could be different from each other. The mixed time delays include time-varying transmission delay, bounded timevarying distributed delay, and constant delay in the leakage term. Firstly, by using the contraction mapping theorem, we obtain a delay-independent sufficient condition to guarantee the global existence and uniqueness of the solution for the addressed neural networks. Secondly, we present a delayindependent sufficient condition to guarantee the existence of an equilibrium point by using topological degree theory. Thirdly, some sufficient conditions which are dependent on the leakage delay, time-varying transmission delay, and distributed delay have been derived to guarantee the global asymptotic stability of the equilibrium point by using a new Lyapunov-Krasovskii functional and some analysis technique. The presented results require neither the boundedness, monotonicity, and differentiability of the activation functions nor the differentiability (even differential boundedness) of time-varying delays, which are more effective and less conservative than other existing literatures [27]. In the absence of leakage delay, the obtained results are also new ones. Moreover, the proposed stability criteria are given in terms of linear matrix inequalities (LMI) [36] and can be conveniently checked by the LMI toolbox in MATLAB. Finally, an example is given to show the effectiveness and less conservativeness of the obtained results.

Notations. Let $\mathbb{R}\left(\mathbb{R}^{+}\right)$denote the set of (positive) real numbers, $\mathbb{Z}_{+}$denote the set of positive integers, and $\mathbb{R}^{n}$ denote the $n$-dimensional real spaces equipped with the Euclidean norm $\|\cdot\| \cdot \mathscr{A}>0$ or $\mathscr{A}<0$ denotes that the matrix $\mathscr{A}$ is a symmetric and positive definite or negative definite matrix. The notations $\mathscr{A}^{T}$ and $\mathscr{A}^{-1}$ mean the transpose of $\mathscr{A}$ and the inverse of a square matrix. $\lambda_{\max }(\mathscr{A})$ or $\lambda_{\text {min }}(\mathscr{A})$ denotes the maximum eigenvalue or the minimum eigenvalue of matrix $\mathscr{A}$. I denotes the identity matrix with appropriate dimensions and $\Lambda=\{1,2, \ldots, n\} .[\cdot]^{*}$ denotes the integer function. For any interval $J \subseteq \mathbb{R}$, set $V \subseteq \mathbb{R}^{k}(1 \leq k \leq n), C(J, V)=$ $\{\varphi: J \rightarrow V$ is continuous $\}$, and $P C^{1}(J, V)=\{\varphi: J \rightarrow$ $V$ is continuously differentiable everywhere except at finite number of points $t$, at which $\varphi\left(t^{+}\right), \varphi\left(t^{-}\right), \dot{\varphi}\left(t^{+}\right)$, and $\dot{\varphi}\left(t^{-}\right)$ exist and $\varphi\left(t^{+}\right)=\varphi(t), \dot{\varphi}\left(t^{+}\right)=\dot{\varphi}(t)$, where $\dot{\varphi}$ denotes the derivative of $\varphi\}$. For any $x=\left(x_{1}, x_{2}, \ldots, x_{n}\right) \in \mathbb{R}^{n}$, $[x]^{+}=\left(\left|x_{1}\right|,\left|x_{2}\right|, \ldots,\left|x_{n}\right|\right)^{T}$ and, for any $Q=\left(q_{i j}\right)_{n \times n} \in \mathbb{Z}^{n \times n}$, $[Q]^{+}=\left(\left|q_{i j}\right|\right)_{n \times n}$. For any $t \in \mathbb{R}^{+}, x_{t}$ is defined by $x_{t}=x(t+s)$, $x_{t^{-}}=x\left(t^{-}+s\right), s \in[-\sigma, 0]$. In addition, the notation $\star$ always denotes the symmetric block in one symmetric matrix.

\section{Preliminaries}

Consider the following impulsive neural networks model:

$$
\begin{aligned}
& \dot{x}(t)=-D x(t-\sigma)+A f(x(t))+B g(x(t-\tau(t))) \\
& \quad+W \int_{t-\rho(t)}^{t} K(t-s) h(x(s)) d s+I, \quad t>0, t \neq t_{k}, \\
& \Delta x\left(t_{k}\right)=x\left(t_{k}\right)-x\left(t_{k}^{-}\right)=I_{k}\left(x\left(t_{k}^{-}\right), x_{t_{k}^{-}}\right), \quad k \in \mathbb{Z}_{+},
\end{aligned}
$$

where $x(t)=\left(x_{1}(t), \ldots, x_{n}(t)\right)^{T}$ is the neuron state vector of the neural network; $D=\operatorname{diag}\left(d_{1}, \ldots, d_{n}\right)$ is a diagonal matrix with $d_{i}>0, i \in \Lambda, \Lambda=\{1,2, \ldots, n\} ; A, B$, and $W$ are the connection weight matrix, the delayed weight matrix, and the distributively delayed connection weight matrix, respectively; $I$ is an external input; $\sigma \geq 0$ is a constant which denotes the leakage delay; $\tau(t)$ is a time-varying transmission delay of the neural network; $\rho(t)$ is a time-varying distributed delay of the neural network; $f(x(\cdot))=\left(f_{1}\left(x_{1}(\cdot)\right), \ldots, f_{n}\left(x_{n}(\cdot)\right)\right)^{T}$, $g(x(\cdot))=\left(g_{1}\left(x_{1}(\cdot)\right), \ldots, g_{n}\left(x_{n}(\cdot)\right)\right)^{T}$, and $h(x(\cdot))=$ $\left(h_{1}\left(x_{1}(\cdot)\right), \ldots, h_{n}\left(x_{n}(\cdot)\right)\right)^{T}$ represent the neuron activation functions; $K(\cdot)=\operatorname{diag}\left(k_{1}(\cdot), \ldots, k_{n}(\cdot)\right)$ is the delay kernel function.

Throughout this paper, we make the following assumptions.

$\left(H_{1}\right) \tau(t)$ represents the discrete transmission delay with $0 \leq \tau(t) \leq \tau ; \rho(t)$ represents the time-varying distributed delay with $0 \leq \rho(t) \leq \rho$, where $\tau, \rho$ are two positive constants.

$\left(H_{2}\right)$ The delay kernels $k_{j}(\cdot), j \in \Lambda$, are some real valued continuous functions defined on $[0, \rho]$ and satisfy

$$
\left|k_{j}(s)\right| \leq|k(s)|, \quad \int_{0}^{\rho}|k(s)| d s=\kappa,
$$

where $\kappa$ is a positive constant.

$\left(H_{3}\right)$ The neuron activation functions $f_{j}, g_{j}$, and $h_{j}, j \in \Lambda$, are continuous on $\mathbb{R}$ and satisfy

$$
\sigma_{j}^{-} \leq \frac{f_{j}(u)-f_{j}(v)}{u-v} \leq \sigma_{j}^{+},
$$




$$
\begin{gathered}
\delta_{j}^{-} \leq \frac{g_{j}(u)-g_{j}(v)}{u-v} \leq \delta_{j}^{+}, \\
\zeta_{j}^{-} \leq \frac{h_{j}(u)-h_{j}(v)}{u-v} \leq \zeta_{j}^{+},
\end{gathered}
$$

for any $u, v \in \mathbb{R}, u \neq v, j \in \Lambda$, where $\sigma_{j}^{-}, \sigma_{j}^{+}, \delta_{j}^{-}, \delta_{j}^{+}$, $\zeta_{j}^{-}$, and $\zeta_{j}^{+}$are some real constants and they may be positive, zero, or negative.

$\left(H_{4}\right) I_{k}(\cdot): \mathbb{R} \times \mathbb{R}^{n} \rightarrow \mathbb{R}^{n}, k \in \mathbb{Z}_{+}$, are some continuous functions.

$\left(H_{5}\right)$ The impulse times $t_{k}$ satisfy $0=t_{0}<t_{1}<\cdots<t_{k} \rightarrow$ $\infty$ and $\inf _{k \in \mathbb{Z}_{+}}\left\{t_{k}-t_{k-1}\right\}>0$.

We will consider model (1) with the initial condition

$$
x(s)=\varphi(s), \quad s \in[-\eta, 0],
$$

where $\eta=\max \{\sigma, \tau, \rho\}, \varphi(\cdot)=\left(\varphi_{1}, \ldots, \varphi_{n}\right)^{T} \in P C^{1}([-\eta, 0])$, $\mathbb{R}^{n}$, whose norm is defined by

$$
\|\varphi\|_{\eta}^{2}=\max \left\{\max _{-\eta \leq \theta \leq 0} \sum_{i=1}^{n}\left|\varphi_{i}^{2}(\theta)\right|, \max _{-\tau \leq \theta \leq 0} \sum_{i=1}^{n}\left|\dot{\varphi}_{i}^{2}(\theta)\right|\right\} .
$$

Definition 1 (see [37]). Assume that $\Omega \in \mathbb{R}^{n}$ is a bounded and open set and $\mathscr{F}(u): \Omega \rightarrow \mathbb{R}^{n}$ is a continuous and differentiable function. If $p \bar{\epsilon} \mathscr{F}(\partial \Omega)$ and $J_{\mathscr{F}}(u) \neq 0$ for any $u \in \mathscr{F}^{-1}(p)$, where $\partial \Omega$ denotes the boundary of $\Omega$ and $J_{\mathscr{F}}$ denotes the Jacobian determinant relative to $\mathscr{F}$, then the topological degree relative to $\Omega$ and $p$ is defined by

$$
\operatorname{deg}(\mathscr{F}, \Omega, p)= \begin{cases}\sum_{u \in \mathscr{F}^{-1}(p)} \operatorname{sgn} J_{\mathscr{F}}(u), & \mathscr{F}^{-1}(p) \neq \emptyset, \\ 0, & \mathscr{F}^{-1}(p)=\emptyset .\end{cases}
$$

Remark 2. Generally speaking, the topological degree of $\mathscr{F}(u)$ relative to $\Omega$ and $p$ can be regarded as the algebraic number of solutions of $\mathscr{F}(u)=p$ in $\Omega$ if $\mathscr{F}(\partial \Omega) \neq 0$. For instance, $\operatorname{deg}(\mathscr{F}, \Omega, 0)= \pm 1$ implies that $\mathscr{F}(u)=0$ has at least one solution in $\Omega$.

Lemma 3 (see [38]). Given any real matrix $M=M^{T}>0$ of appropriate dimension and a vector function $\omega(\cdot):[a, b] \rightarrow$ $\mathbb{R}^{n}$, such that the integrations concerned are well defined, then

$$
\begin{aligned}
& {\left[\int_{a}^{b} \omega(s) d s\right]^{T} M\left[\int_{a}^{b} \omega(s) d s\right]} \\
& \quad \leq(b-a) \int_{a}^{b} \omega^{T}(s) M \omega(s) d s .
\end{aligned}
$$

Lemma 4 (see [12]). Given any real matrices $\Sigma_{1}, \Sigma_{2}, \Sigma_{3}$ of appropriate dimensions and a scalar $\epsilon>0$ such that $0<\Sigma_{3}=$ $\Sigma_{3}^{T}$, then the following inequality holds:

$$
\Sigma_{1}^{T} \Sigma_{2}+\Sigma_{2}^{T} \Sigma_{1} \leq \epsilon \Sigma_{1}^{T} \Sigma_{3} \Sigma_{1}+\epsilon^{-1} \Sigma_{2}^{T} \Sigma_{3}^{-1} \Sigma_{2}
$$

Lemma 5 (see [27]). Supposing that $\mho, \mho_{i j} \geq 0(i, j=1,2)$ are symmetric matrices of appropriate dimensions, $\alpha \in[0,1]$ and $\beta \in[0,1]$, then $\mho+\left[(1-\alpha) \mho_{11}+\alpha \mho_{12}\right]+\left[(1-\beta) \mho_{21}+\right.$ $\left.\beta \mho_{22}\right]<0$ holds if the following four inequalities $\mho+\mho_{11}+$ $\mho_{21}<0, \mho+\mho_{11}+\mho_{22}<0, \mho+\mho_{12}+\mho_{21}<0$, and $\mho+\mho_{12}+$ $\mho_{22}<0$ hold simultaneously.

For presentation convenience, in the following, we denote

$$
\begin{aligned}
& \Sigma_{1}=\operatorname{diag}\left(\sigma_{1}^{-} \sigma_{1}^{+}, \ldots, \sigma_{n}^{-} \sigma_{n}^{+}\right), \\
& \Sigma_{2}=\operatorname{diag}\left(\frac{\sigma_{1}^{-}+\sigma_{1}^{+}}{2}, \ldots, \frac{\sigma_{n}^{-}+\sigma_{n}^{+}}{2}\right), \\
& \Sigma_{3}=\operatorname{diag}\left(\delta_{1}^{-} \delta_{1}^{+}, \ldots, \delta_{n}^{-} \delta_{n}^{+}\right), \\
& \Sigma_{4}=\operatorname{diag}\left(\frac{\delta_{1}^{-}+\delta_{1}^{+}}{2}, \ldots, \frac{\delta_{n}^{-}+\delta_{n}^{+}}{2}\right), \\
& \Sigma_{5}=\operatorname{diag}\left(\zeta_{1}^{-} \zeta_{1}^{+}, \ldots, \zeta_{n}^{-} \zeta_{n}^{+}\right), \\
& \Sigma_{6}=\operatorname{diag}\left(\frac{\zeta_{1}^{-}+\zeta_{1}^{+}}{2}, \ldots, \frac{\zeta_{n}^{-}+\zeta_{n}^{+}}{2}\right) .
\end{aligned}
$$

\section{Global Existence and Uniqueness of Solution}

In this section, by using the contraction mapping theorem, we give a delay-independent sufficient condition to guarantee the global existence and uniqueness of the solution for models (1) and (4).

Theorem 6. Assume that the assumptions $\left(H_{1}\right)-\left(H_{5}\right)$ hold; then the solution $x=x(t, 0, \varphi)$ of models (1) and (4) exists uniquely on $[-\eta, \infty)$.

Proof. Transform the global existence and uniqueness of solution of the models (1) and (4) into a fixed point problem. Let $\|\cdot\|^{*}$ be the norm in $C\left(\left[0, t_{1}\right], \mathbb{R}^{n}\right)$ defined by

$$
\|u\|^{*} \doteq \max _{t \in\left[0, t_{1}\right]}\left\{e^{-\lambda t} \cdot \max _{s \in[0, t]}\{\|u(s)\|\}\right\}, \quad u \in C\left(\left[0, t_{1}\right], \mathbb{R}^{n}\right)
$$

where

$$
\begin{gathered}
\lambda=\sqrt{\sum_{j=1}^{n} d_{j}^{2}}+\sigma \cdot \sqrt{\sum_{i=1}^{n} \sum_{j=1}^{n} a_{i j}^{2}}+\delta \cdot \sqrt{\sum_{i=1}^{n} \sum_{j=1}^{n} b_{i j}^{2}} \\
+\zeta \cdot \kappa \cdot \sqrt{\sum_{i=1}^{n} \sum_{j=1}^{n} w_{i j}^{2}+1,} \\
\sigma=\max _{j \in \Lambda}\left\{\left|\sigma_{j}^{-}\right|,\left|\sigma_{j}^{+}\right|\right\}, \quad \delta=\max _{j \in \Lambda}\left\{\left|\delta_{j}^{-}\right|,\left|\delta_{j}^{+}\right|\right\}, \\
\zeta=\max _{j \in \Lambda}\left\{\left|\zeta_{j}^{-}\right|,\left|\zeta_{j}^{+}\right|\right\} .
\end{gathered}
$$

Then it is easy to see that $C\left(\left[0, t_{1}\right], \mathbb{R}^{n}\right)$ is a Banach space endowed with the norm $\|\cdot\|^{*}$. Let $u \in C\left(\left[0, t_{1}\right], \mathbb{R}^{n}\right)$ and 
consider the operator $\mathscr{L}_{1}: C\left(\left[0, t_{1}\right], \mathbb{R}^{n}\right) \rightarrow C\left(\left[0, t_{1}\right], \mathbb{R}^{n}\right)$ defined by

$$
\begin{aligned}
& \left(\mathscr{L}_{1} u\right)(t) \\
& =\varphi(0)+\int_{0}^{t}\{-D u(s-\sigma)+A f(u(s)) \\
& +B g(u(s-\tau(s))) \\
& \left.+W \int_{0}^{\rho(t)} K(\theta) h(u(s-\theta)) d \theta+I\right\} d s,
\end{aligned}
$$

where $u(s)=\varphi(s), s \in[-\eta, 0]$.

First we show that $\mathscr{L}_{1}$ is a contraction on $C\left(\left[0, t_{1}\right], \mathbb{R}^{n}\right)$. Let $u, v \in C\left(\left[0, t_{1}\right], \mathbb{R}^{n}\right)$; we have

$$
\begin{aligned}
& \left\|\left(\mathscr{L}_{1} u\right)(t)-\left(\mathscr{L}_{1} v\right)(t)\right\| \\
& =\|-\int_{0}^{t} D[u(s-\sigma)-v(s-\sigma)] d s \\
& +\int_{0}^{t} A[f(u(s))-f(v(s))] d s \\
& +\int_{0}^{t} B[f(u(s-\tau(s)))-f(v(s-\tau(s)))] d s \\
& +\int_{0}^{t} W \int_{0}^{\rho(t)} K(\theta)[h(u(s-\theta))-h(v(s-\theta))] d \theta d s \| \\
& \leq \int_{0}^{t}\|D[u(s-\sigma)-v(s-\sigma)]\| d s \\
& +\int_{0}^{t}\|A[f(u(s))-f(v(s))]\| d s \\
& +\int_{0}^{t}\|B[f(u(s-\tau(s)))-f(v(s-\tau(s)))]\| d s \\
& +\int_{0}^{t}\left\|W \int_{0}^{\rho(t)} K(\theta)[h(u(s-\theta))-h(v(s-\theta))] d \theta\right\| d s \\
& \leq \sqrt{\sum_{j=1}^{n} d_{j}^{2}} \int_{0}^{t}\|u(s-\sigma)-v(s-\sigma)\| d s \\
& +\sigma \cdot \sqrt{\sum_{i=1}^{n} \sum_{j=1}^{n} a_{i j}^{2}} \int_{0}^{t}\|u(s)-v(s)\| d s \\
& +\delta \cdot \sqrt{\sum_{i=1}^{n} \sum_{j=1}^{n} b_{i j}^{2}} \int_{0}^{t}\|u(s-\tau(s))-v(s-\tau(s))\| d s \\
& +\int_{0}^{t}\left\|W \int_{0}^{\rho(t)} K(\theta)[h(u(s-\theta))-h(v(s-\theta))] d \theta\right\| d s .
\end{aligned}
$$

In view of $\left(\mathrm{H}_{2}\right)$, we get

$$
\begin{aligned}
& \int_{0}^{t}\left\|W \int_{0}^{\rho(t)} K(\theta)[h(u(s-\theta))-h(v(s-\theta))] d \theta\right\| d s \\
& \quad \leq \zeta \cdot \sqrt{\sum_{i=1}^{n} \sum_{j=1}^{n} w_{i j}^{2}} \int_{0}^{t}\left\|\int_{0}^{\rho(t)} K(\theta)[u(s-\theta)-v(s-\theta)] d \theta\right\| d s \\
& \quad \leq \zeta \cdot \sqrt{\sum_{i=1}^{n} \sum_{j=1}^{n} w_{i j}^{2}} \int_{0}^{t} \int_{0}^{\rho(t)}\|K(\theta)[u(s-\theta)-v(s-\theta)]\| d \theta d s \\
& \quad \leq \zeta \cdot \sqrt{\sum_{i=1}^{n} \sum_{j=1}^{n} w_{i j}^{2}} \int_{0}^{t} \int_{0}^{\rho(t)}|k(\theta)|\|u(s-\theta)-v(s-\theta)\| d \theta d s \\
& \quad \leq \zeta \cdot \sqrt{\sum_{i=1}^{n} \sum_{j=1}^{n} w_{i j}^{2}} \int_{0}^{t} \int_{0}^{\rho(t)}|k(\theta)| d \theta \max _{r \in[0, s]}\|u(r)-v(r)\| d s \\
& \quad \leq \zeta \cdot \kappa \cdot \sqrt{\sum_{i=1}^{n} \sum_{j=1}^{n} w_{i j}^{2}} \int_{0}^{t} \max _{r \in[0, s]}\|u(r)-v(r)\| d s .
\end{aligned}
$$

Substituting the above inequality to (14), we obtain

$$
\begin{aligned}
&\left\|\left(\mathscr{L}_{1} u\right)(t)-\left(\mathscr{L}_{1} v\right)(t)\right\| \\
& \leq \sqrt{\sum_{j=1}^{n} d_{j}^{2}} \int_{0}^{t} \max _{r \in[0, s]}\|u(r)-v(r)\| d s \\
&+\sigma \cdot \sqrt{\sum_{i=1}^{n} \sum_{j=1}^{n} a_{i j}^{2}} \int_{0}^{t} \max _{r \in[0, s]}\|u(r)-v(r)\| d s \\
&+\delta \cdot \sqrt{\sum_{i=1}^{n} \sum_{j=1}^{n} b_{i j}^{2}} \int_{0}^{t} \max _{r \in[0, s]}\|u(r)-v(r)\| d s \\
&+\zeta \cdot \kappa \cdot \sqrt{\sum_{i=1}^{n} \sum_{j=1}^{n} w_{i j}^{2}} \int_{0}^{t} \max _{r \in[0, s]}^{t}\|u(r)-v(r)\| d s \\
&=(\lambda-1) \int_{0}^{t} \max _{r \in[0, s]}\|u(r)-v(r)\| d s \\
&=(\lambda-1) \int_{0}^{t} e^{\lambda s} e^{-\lambda s} \max _{r \in[0, s]}\|u(r)-v(r)\| d s \\
& \leq(\lambda-1) \int_{0}^{t} e^{\lambda d s} \max _{s \in\left[0, t_{1}\right]}\left\{e^{-\lambda s} \max _{r \in[0, s]}\|u(r)-v(r)\|\right\} d s \\
& \leq \frac{\lambda-1}{\lambda} e^{\lambda t}\|u-v\|^{*} . \\
& \int_{0}^{t} e^{\lambda s} d s\|u-v\|^{*}
\end{aligned}
$$


Since $e^{\lambda t}$ is increasing in $t$,

$$
\max _{s \in[0, t]}\left\{\left\|\left(\mathscr{L}_{1} u\right)(s)-\left(\mathscr{L}_{1} v\right)(s)\right\|\right\} \leq \frac{\lambda-1}{\lambda} e^{\lambda t}\|u-v\|^{*} .
$$

Then

$$
e^{-\lambda t} \max _{s \in[0, t]}\left\{\left\|\left(\mathscr{L}_{1} u\right)(s)-\left(\mathscr{L}_{1} v\right)(s)\right\|\right\} \leq \frac{\lambda-1}{\lambda}\|u-v\|^{*} .
$$

Note the definition of $\|\cdot\|^{*}$; we have

$$
\left\|\mathscr{L}_{1} u-\mathscr{L}_{1} v\right\|^{*} \leq \frac{\lambda-1}{\lambda}\|u-v\|^{*} .
$$

Thus $\mathscr{L}_{1}$ is a contraction on $C\left(\left[0, t_{1}\right], \mathbb{R}^{n}\right)$, and it has a unique fixed point $u_{1}^{*} \in C\left(\left[0, t_{1}\right], \mathbb{R}^{n}\right)$. Thus we get the fact that $u_{1}^{*}\left(t_{1}\right)$ exists finitely. It implies that $u_{1}^{*}\left(t_{1}\right)+J_{1}\left(u_{1}^{*}\left(t_{1}\right), u_{1 t_{1}}^{*}\right)$ also exists finitely, since assumption $\left(H_{4}\right)$ holds. Then we replace $u_{1}^{*}\left(t_{1}\right)$ with $u_{1}^{*}\left(t_{1}\right)+I_{1}\left(u_{1}^{*}\left(t_{1}\right), u_{1 t_{1}}^{*}\right)$ and define $\eta_{1}=$ $u_{1}^{*}\left(t_{1}\right)+I_{1}\left(u_{1}^{*}\left(t_{1}\right), u_{1 t_{1}}^{*}\right)$ for later use.

Next we show that $\mathscr{L}_{2}$ is a contraction on $C\left(\left[t_{1}, t_{2}\right], \mathbb{R}^{n}\right)$. For $u \in C\left(\left[t_{1}, t_{2}\right], \mathbb{R}^{n}\right)$, let

$$
\|u\|^{*} \doteq \max _{t \in\left[t_{1}, t_{2}\right]}\left\{e^{-\lambda\left(t-t_{1}\right)} \cdot \max _{s \in\left[t_{1}, t\right]}\{\|u(s)\|\}\right\},
$$

where $\lambda$ is defined in (11). Let $u \in C\left(\left[t_{1}, t_{2}\right], \mathbb{R}^{n}\right)$ and consider the operator $\mathscr{L}_{2}: C\left(\left[t_{1}, t_{2}\right], \mathbb{R}^{n}\right) \rightarrow C\left(\left[t_{1}, t_{2}\right], \mathbb{R}^{n}\right)$ defined by

$$
\begin{aligned}
& \left(\mathscr{L}_{2} u\right)(t) \\
& =\eta_{1}+\int_{t_{1}}^{t}\{-D u(s-\sigma)+A f(u(s)) \\
& +B g(u(s-\tau(s))) \\
& \left.+W \int_{0}^{\rho(t)} K(\theta) h(u(s-\theta)) d \theta+I\right\} d s
\end{aligned}
$$

where $\eta_{1}=u_{1}^{*}\left(t_{1}\right)+I_{1}\left(u_{1}^{*}\left(t_{1}\right)\right)$ and

$$
u(s)= \begin{cases}\varphi(s), & s \in[-\eta, 0] \\ u_{1}^{*}(s), & s \in\left[0, t_{1}\right) .\end{cases}
$$

By virtue of the definition of $\mathscr{L}_{2}$, similar to the proof of (19), we get, for $u, v \in C\left(\left[t_{1}, t_{2}\right], \mathbb{R}^{n}\right)$,

$$
\left\|\mathscr{L}_{2} u-\mathscr{L}_{2} v\right\|^{*} \leq \frac{\lambda-1}{\lambda}\|u-v\|^{*} .
$$

Thus $\mathscr{L}_{2}$ is a contraction on $C\left(\left[t_{1}, t_{2}\right], \mathbb{R}^{n}\right)$, and it has a unique fixed point $u_{2}^{*} \in C\left(\left[t_{1}, t_{2}\right], \mathbb{R}^{n}\right)$. Moreover, we know that $u_{2}^{*}\left(t_{2}\right)$ exists finitely, which implies that $u_{2}^{*}\left(t_{2}\right)+$ $I_{2}\left(u_{2}^{*}\left(t_{2}\right), u_{2 t_{2}}^{*}\right)$ exists finitely in view of assumption $\left(H_{4}\right)$. Then we replace $u_{2}^{*}\left(t_{2}\right)$ with $u_{2}^{*}\left(t_{2}\right)+I_{2}\left(u_{2}^{*}\left(t_{2}\right), u_{2 t_{2}}^{*}\right)$ and define $\eta_{2}=u_{2}^{*}\left(t_{2}\right)+I_{2}\left(u_{2}^{*}\left(t_{2}\right), u_{2 t_{2}}^{*}\right)$ for later use.
Finally we show that $\mathscr{L}_{n+1}$ is a contraction on $C\left(\left[t_{n}, t_{n+1}\right], \mathbb{R}^{n}\right)$. For $u \in C\left(\left[t_{n}, t_{n+1}\right], \mathbb{R}^{n}\right)$, let

$$
\|u\|^{*} \doteq \max _{t \in\left[t_{n}, t_{n+1}\right]}\left\{e^{-\lambda\left(t-t_{n}\right)} \cdot \max _{s \in\left[t_{n}, t\right]}\{\|u(s)\|\}\right\},
$$

where $\lambda$ is defined in (11). Let $u \in C\left(\left[t_{n}, t_{n+1}\right], \mathbb{R}^{n}\right)$; then we can similarly consider the operator $\mathscr{L}_{n+1}$ : $C\left(\left[t_{n}, t_{n+1}\right], \mathbb{R}^{n}\right) \rightarrow C\left(\left[t_{n}, t_{n+1}\right], \mathbb{R}^{n}\right)$ defined by

$$
\begin{aligned}
\left(\mathscr{L}_{n+1} u\right)(t) & \\
=\eta_{n}+\int_{t_{n}}^{t}\{ & -D u(s-\sigma)+A f(u(s)) \\
& +B g(u(s-\tau(s))) \\
& \left.+W \int_{0}^{\rho(t)} K(\theta) h(u(s-\theta)) d \theta+I\right\} d s,
\end{aligned}
$$

where $\eta_{n}=u_{n}^{*}\left(t_{n}\right)+I_{n}\left(u_{n}^{*}\left(t_{n}\right)\right)$ and

$$
u(s)= \begin{cases}\varphi(s), & s \in[-\eta, 0], \\ u_{1}^{*}(s), & s \in\left[0, t_{1}\right), \\ u_{2}^{*}(s), & s \in\left[t_{1}, t_{2}\right), \\ \vdots & \\ u_{n}^{*}(s), & s \in\left[t_{n-1}, t_{n}\right) .\end{cases}
$$

Then repeating the argument with $\mathscr{L}_{n+1}$ replacing $\mathscr{L}_{1}$, similar to the proof of (19), we see that, for $u, v \in C\left(\left[t_{n}, t_{n+1}\right], \mathbb{R}^{n}\right)$,

$$
\left\|\mathscr{L}_{n+1} u-\mathscr{L}_{n+1} v\right\|^{*} \leq \frac{\lambda-1}{\lambda}\|u-v\|^{*} .
$$

Thus $\mathscr{L}_{n+1}$ is a contraction on $C\left(\left[t_{n}, t_{n+1}\right], \mathbb{R}^{n}\right)$, and it has a unique fixed point $u_{n+1}^{*} \in C\left(\left[t_{n}, t_{n+1}\right], \mathbb{R}^{n}\right)$.

Continuing in this manner, we construct

$$
u^{*}(t)= \begin{cases}\varphi(t), & t \in[-\eta, 0], \\ u_{1}^{*}(t), & t \in\left[0, t_{1}\right), \\ u_{2}^{*}(t), & t \in\left[t_{1}, t_{2}\right), \\ \vdots & \\ u_{n+1}^{*}(t), & t \in\left[t_{n}, t_{n+1}\right), \\ \vdots & \end{cases}
$$

Then $u^{*}(t)$ is the global solution of models (1) and (4). If $v^{*}(t)$ is another solution of models (1) and (4), then it is easy to check from the above argument that $u^{*}(t)=v^{*}(t)$. Hence, the solution $u^{*}(t)=u^{*}(t, 0, \varphi)$ of models (1) and (4) exists uniquely on $[-\eta, \infty)$. This completes the proof.

\section{Existence of an Equilibrium Point}

In previous sections, we have showed the global existence and uniqueness of solution for models (1) and (4). In this section, without requiring the boundedness, differentiability, 
or monotonicity of the activation functions, we establish a delay-independent sufficient condition for the existence of an equilibrium point of model (1). As usual, we denote an equilibrium point of the model (1) by the constant vector $x^{*} \in \mathbb{R}^{n}$, where $x^{*}$ satisfies

$$
\begin{gathered}
-D x^{*}+A f\left(x^{*}\right)+B g\left(x^{*}\right)+W \mathbb{K} h\left(x^{*}\right)+I=0, \\
I_{k}\left(x^{*}, x^{*}\right)=0, \quad k \in \mathbb{Z}_{+},
\end{gathered}
$$

where $\mathbb{K}=\mathbb{K}(t)=\int_{0}^{\rho(t)} K(s) d s$. In this paper, it is assumed that the impulsive function $I_{k}$ satisfies $I_{k}\left(x^{*}, x^{*}\right)=0$ for $k \in \mathbb{Z}_{+}$. Hence, to prove the existence of solution of (29), it suffices to show that the following has a solution:

$$
\begin{aligned}
x^{*}- & D^{-1} A f\left(x^{*}\right)-D^{-1} B g\left(x^{*}\right) \\
- & D^{-1} W \mathbb{K} h\left(x^{*}\right)-D^{-1} I=0,
\end{aligned}
$$

in view of $D>0$.

Theorem 7. Assume that the assumption $\left(H_{3}\right)$ holds. Then model (1) has at least one equilibrium point if, for any $t>0$, $D-[A]^{+} \Sigma-[B]^{+} \Delta-[W \mathbb{K}]^{+} \Theta$ is an $M$-matrix, where $\Sigma=$ $\operatorname{diag}\left(\sigma_{1}, \ldots, \sigma_{n}\right), \sigma_{j}=\max \left\{\left|\sigma_{j}^{-}\right|,\left|\sigma_{j}^{+}\right|\right\}, \Delta=\operatorname{diag}\left(\delta_{1}, \ldots, \delta_{n}\right)$, $\delta_{j}=\max \left\{\left|\delta_{j}^{-}\right|,\left|\delta_{j}^{+}\right|\right\}$, and $\Theta=\operatorname{diag}\left(\zeta_{1}, \ldots, \zeta_{n}\right), \zeta_{j}=$ $\max \left\{\left|\zeta_{j}^{-}\right|,\left|\zeta_{j}^{+}\right|\right\}$.

Proof. From (30), we note that it suffices to prove that the following has at least one solution:

$$
p(x)=x-W_{1} f(x)-W_{2} g(x)-W_{3} h(x)-J^{\prime}=0,
$$

where $W_{1}=D^{-1} A, W_{2}=D^{-1} B, W_{3}=D^{-1} W \mathbb{K}, J^{\prime}=D^{-1} J$. In order to use topological degree theory, we consider the following homotopic mapping:

$$
P(x, \lambda)=\lambda p(x)+(1-\lambda) x, \quad \lambda \in[0,1] .
$$

Note that $D-[A]^{+} \Sigma-[B]^{+} \Delta-[W \mathbb{K}]^{+} \Theta$ is an $M$-matrix; it can be deduced that $I-\left[W_{1}\right]^{+} \Sigma-\left[W_{2}\right]^{+} \Delta-\left[W_{3}\right]^{+} \Theta$ is also an $M-$ matrix. This implies that $\left(I-\left[W_{1}\right]^{+} \Sigma-\left[W_{2}\right]^{+} \Delta-\left[W_{3}\right]^{+} \Theta\right)^{-1} \geq$ 0 and there exists a positive vector $X_{0} \in \mathbb{R}^{n}$ such that (I$\left.\left[W_{1}\right]^{+} \Sigma-\left[W_{2}\right]^{+} \Delta-\left[W_{3}\right]^{+} \Theta\right) X_{0}>0$. It then follows that

$$
\begin{aligned}
{[P(x, \lambda)]^{+} } \\
=[\lambda p(x)+(1-\lambda) x]^{+} \\
=\left[x-\lambda W_{1} f(x)-\lambda W_{2} g(x)-\lambda W_{3} h(x)-\lambda J^{\prime}\right]^{+} \\
\geq[x]^{+}-\lambda\left[W_{1} f(x)\right]^{+}-\lambda\left[W_{2} g(x)\right]^{+} \\
\quad-\lambda\left[W_{3} h(x)\right]^{+}-\lambda\left[J^{\prime}\right]^{+}
\end{aligned}
$$

$$
\begin{aligned}
\geq & {[x]^{+}-\lambda\left[W_{1}\right]^{+}[f(x)]^{+}-\lambda\left[W_{2}\right]^{+}[g(x)]^{+} } \\
& -\lambda\left[W_{3}\right]^{+}[h(x)]^{+}-\lambda\left[J^{\prime}\right]^{+} \\
\geq & {[x]^{+}-\lambda\left[W_{1}\right]^{+} \Sigma[x]^{+}-\lambda\left[W_{2}\right]^{+} \Delta[x]^{+} } \\
& -\lambda\left[W_{3}\right]^{+} \Theta[x]^{+}-\lambda\left[J^{\prime}\right]^{+} \\
\geq & (1-\lambda)[x]^{+}+\lambda\left(I-\left[W_{1}\right]^{+} \Sigma-\left[W_{2}\right]^{+} \Delta-\left[W_{3}\right]^{+} \Theta\right) \\
& \cdot\left\{[x]^{+}-\left(I-\left[W_{1}\right]^{+} \Sigma-\left[W_{2}\right]^{+} \Delta-\left[W_{3}\right]^{+} \Theta\right)^{-1}\left[J^{\prime}\right]^{+}\right\} .
\end{aligned}
$$

Let

$$
\begin{aligned}
\Omega=\left\{x \mid[x]^{+} \leq\right. & \left(I-\left[W_{1}\right]^{+} \Sigma-\left[W_{2}\right]^{+} \Delta\right. \\
& \left.\left.-\left[W_{3}\right]^{+} \Theta\right)^{-1}\left[J^{\prime}\right]^{+}+X_{0}\right\} .
\end{aligned}
$$

It is obvious that set $\Omega$ is not empty and, for any $x \in \partial \Omega$, we have

$$
\begin{aligned}
{[P(x, \lambda)]^{+} \geq } & (1-\lambda)[x]^{+} \\
& +\lambda\left(I-\left[W_{1}\right]^{+} \Sigma-\left[W_{2}\right]^{+} \Delta-\left[W_{3}\right]^{+} \Theta\right) X_{0} \\
> & 0, \quad \lambda \in[0,1]
\end{aligned}
$$

which implies that $P(x, \lambda) \neq 0$ for all $x \in \partial \Omega$ and $\lambda \in[0,1]$. By topological degree invariance theory, we obtain

$$
\begin{aligned}
\operatorname{deg}(p(x), \Omega, 0) & =\operatorname{deg}(P(x, \lambda), \Omega, 0) \\
& =\operatorname{deg}(P(x, 0), \Omega, 0)=1 .
\end{aligned}
$$

Therefore, from Remark 2 , we know that $p(x)=0$ has at least one solution in $\Omega$. This completes the proof.

\section{Global Asymptotic Stability}

It should be noted that Theorem 7 can guarantee the existence of an equilibrium point but not the uniqueness. In this section, we will derive some sufficient conditions to guarantee not only the global asymptotic stability but also the uniqueness of the equilibrium point. For this purpose, the impulsive function $I_{k}$ which is viewed as a perturbation of the equilibrium point $x^{*}$ of models (1) and (4) without impulses is defined by

$$
\begin{aligned}
x( & \left.t_{k}\right)-x\left(t_{k}^{-}\right) \\
& =I_{k}\left(x\left(t_{k}^{-}\right), x_{t_{k}^{-}}\right) \\
& =-J_{k}\left\{x\left(t_{k}^{-}\right)-x^{*}-D \int_{t_{k}-\sigma}^{t_{k}}\left(x(u)-x^{*}\right) d u\right\}, \quad k \in \mathbb{Z}_{+},
\end{aligned}
$$

where $J_{k}, k \in \mathbb{Z}_{+}$are some $n \times n$ real matrices. It is clear that $I_{k}\left(x^{*}, x^{*}\right)=0, k \in \mathbb{Z}_{+}$. Such type of impulses describes 
the fact that the encountered instantaneous perturbations depend on not only the state of neurons at impulse times $t_{k}$ but also the state of neurons in its recent history, which reflects more realistic dynamics. The similar nonlinear impulsive perturbations, which include linear impulsive perturbations and nonimpulsive perturbations as their special cases, have also been investigated by some researchers recently [17$21,39]$.

Let $y(t)=x(t)-x^{*}$; then we rewrite the models (1) and (4) as follows:

$$
\begin{aligned}
\dot{y}(t)= & -D y(t-\sigma)+A \widehat{f}(y(t))+B \widehat{g}(y(t-\tau(t))) \\
& +W \int_{t-\rho(t)}^{t} K(t-s) \widehat{h}(y(s)) d s, \\
\Delta y\left(t_{k}\right)= & y\left(t_{k}\right)-y\left(t_{k}^{-}\right) \\
& =-J_{k}\left\{y\left(t_{k}^{-}\right)-D \int_{t_{k}-\sigma}^{t_{k}} y(u) d u\right\}, \quad t \neq t_{k}, \\
y(s)= & \varphi(s)-x^{*}, \quad s \in[-\eta, 0],
\end{aligned}
$$

where $\widehat{f}(y(\cdot))=f\left(y(\cdot)+x^{*}\right)-f\left(x^{*}\right), \widehat{g}(y(\cdot))=g\left(y(\cdot)+x^{*}\right)-$ $g\left(x^{*}\right)$ and $\widehat{h}(y(\cdot))=h\left(y(\cdot)+x^{*}\right)-h\left(x^{*}\right)$. For convenience in our discussion, in the following, we replace $\hat{f}$ with $f$, replace $\widehat{g}$ with $g$, and replace $\widehat{h}$ with $h$. Then using a simple transformation, model (38) has an equivalent form as follows:

$$
\begin{aligned}
& \frac{d}{d t}\left[y(t)-D \int_{t-\sigma}^{t} y(u) d u\right] \\
& \quad=-D y(t)+A f(y(t))+B g(y(t-\tau(t)))
\end{aligned}
$$

$$
\begin{aligned}
&+W \int_{t-\rho(t)}^{t} K(t-s) h(y(s)) d s, \quad t>0, t \neq t_{k}, \\
& \Delta y\left(t_{k}\right)= y\left(t_{k}\right)-y\left(t_{k}^{-}\right) \\
&=-J_{k}\left\{y\left(t_{k}^{-}\right)-D \int_{t_{k}-\sigma}^{t_{k}} y(u) d u\right\}, \quad k \in \mathbb{Z}_{+}, \\
& y(s)=\varphi(s)-x^{*}, \quad s \in[-\eta, 0] .
\end{aligned}
$$

Theorem 8. Under the conditions in Theorem 7, model (1) has a unique equilibrium point which is globally asymptotically stable if there exist a constant $\gamma>0$, an $n \times n$ inverse matrix $Q_{1}$, six $n \times n$ matrices $P>0, Q_{2}>0, Q_{3}>0, Z>0, T_{11}>0$, and $T_{22}>0$, three $n \times n$ diagonal matrices $U_{1}>0, U_{2}>0$, and $U_{3}>0$, and real matrices $T_{12}, X_{l}(l=1, \ldots, 6)$ with appropriate dimension such that

$$
\begin{gathered}
{\left[\begin{array}{cc}
T_{11} & T_{12} \\
\star & T_{22}
\end{array}\right]>0,} \\
{\left[\begin{array}{cc}
P\left(I-J_{k}\right)^{T} P \\
\star & P
\end{array}\right] \geq 0, \quad k \in \mathbb{Z}_{+},} \\
{\left[\begin{array}{cc}
\Xi & \chi_{j} \\
\star & -Z
\end{array}\right]<0, \quad j=1,2,}
\end{gathered}
$$

where

$$
\begin{aligned}
& \Xi=\left[\begin{array}{cccccccccc}
\Pi_{11} & -Q_{1} & \gamma T_{12}^{T} & -Q_{1} D & X_{1} & D P D & \Pi_{17} & \Pi_{18} & U_{3} \Sigma_{6} & \Pi_{1,10} \\
\star & \Pi_{22} & 0 & -\gamma Q_{1} D & X_{2} & 0 & \gamma Q_{1} A & \gamma Q_{1} B & 0 & \Pi_{2,10} \\
\star & \star & \Pi_{33} & 0 & 0 & 0 & 0 & U_{2} \Sigma_{4} & 0 & 0 \\
\star & \star & \star & -Q_{3} & 0 & 0 & 0 & 0 & 0 & 0 \\
\star & \star & \star & \star & \Pi_{55} & 0 & 0 & 0 & 0 & 0 \\
\star & \star & \star & \star & \star & -Q_{2} & -D P A & -D P B & 0 & -D P W \\
\star & \star & \star & \star & \star & \star & -U_{1} & 0 & 0 & 0 \\
\star & \star & \star & \star & \star & \star & \star & -U_{2} & 0 & 0 \\
\star & \star & \star & \star & \star & \star & \star & \star & \Pi_{99} & 0 \\
\star & \star & \star & \star & \star & \star & \star & \star & \star & \Pi_{10,10}
\end{array}\right], \\
& \chi_{1}=\operatorname{col}\left\{X_{1}, X_{2}, O, O, X_{3}, O, O, O, O, O\right\} \text {, } \\
& \chi_{2}=\operatorname{col}\left\{X_{4}, X_{5}, O, O, O, O, O, O, O, X_{6}\right\} \text {, }
\end{aligned}
$$

with

$$
\begin{aligned}
& \Pi_{11}=-P D-D P+\sigma^{2} Q_{2}+Q_{3}-U_{1} \Sigma_{1}-U_{3} \Sigma_{5}, \\
& \Pi_{17}=P A+Q_{1} A+U_{1} \Sigma_{2}, \\
& \Pi_{18}=P B+Q_{1} B, \\
& \Pi_{1,10}=P W+Q_{1} W+X_{4}, \\
& \Pi_{22}=-\gamma Q_{1}-\gamma Q_{1}^{T}+\gamma^{2} \tau T_{22},
\end{aligned}
$$

$$
\begin{aligned}
& \Pi_{2,10}=\gamma Q_{1} W+X_{5}, \\
& \Pi_{33}=\tau T_{11}-\gamma T_{12}^{T}-\gamma T_{12}-U_{2} \Sigma_{3}, \\
& \Pi_{55}=X_{3}+X_{3}^{T}, \\
& \Pi_{99}=\rho^{2} K(0) Z K(0)-U_{3}, \\
& \Pi_{10,10}=X_{6}+X_{6}^{T} .
\end{aligned}
$$


Proof. Consider the following Lyapunov-Krasovskii functional as

$$
\begin{aligned}
V(t, y(t))= & V_{1}(t, y(t))+V_{2}(t, y(t))+V_{3}(t, y(t)) \\
& +V_{4}(t, y(t))+V_{5}(t, y(t)),
\end{aligned}
$$

where

$$
\begin{aligned}
V_{1}(t, y(t))= & {\left[y(t)-D \int_{t-\sigma}^{t} y(u) d u\right]^{T} } \\
& \times P\left[y(t)-D \int_{t-\sigma}^{t} y(u) d u\right],
\end{aligned}
$$$$
V_{2}(t, y(t))=\sigma \int_{t-\sigma}^{t} \int_{s}^{t} y^{T}(u) Q_{2} y(u) d u d s
$$$$
+\int_{t-\sigma}^{t} y^{T}(s) Q_{3} y(s) d s,
$$$$
V_{3}(t, y(t))=\int_{0}^{t} \int_{u-\tau(u)}^{u}\left(\begin{array}{c}
y(u-\tau(u)) \\
\gamma \dot{y}(s)
\end{array}\right)^{T}\left(\begin{array}{cc}
T_{11} & T_{12} \\
\star & T_{22}
\end{array}\right)
$$$$
\times\left(\begin{array}{c}
y(u-\tau(u)) \\
\gamma \dot{y}(s)
\end{array}\right) d s d u
$$$$
V_{4}(t, y(t))=\gamma^{2} \int_{-\tau}^{0} \int_{t+u}^{t} \dot{y}^{T}(s) T_{22} \dot{y}(s) d s d u,
$$$$
V_{5}(t, y(t))=\rho \int_{t-\rho}^{t} \int_{u}^{t} h^{T}(y(s)) K(t-s) Z K(t-s)
$$

$$
\times h(y(s)) d s d u \text {. }
$$

Calculating the upper right derivative of $V$ along the trajectories of model (39) at the continuous interval $\left[t_{k-1}, t_{k}\right), k \in \mathbb{Z}_{+}$, we obtain

$$
\begin{aligned}
D^{+} V_{1}= & 2\left[y(t)-D \int_{t-\sigma}^{t} y(u) d u\right]^{T} \\
& \times P[-D y(t)+A f(y(t))+B g(y(t-\tau(t))) \\
& \left.+W \int_{t-\rho(t)}^{t} K(t-s) h(y(s)) d s\right] \\
= & -2 y^{T}(t) P D y(t)+2 y^{T}(t) P A f(y(t)) \\
& +2 y^{T}(t) P B g(y(t-\tau(t))) \\
& +2 y^{T}(t) P W \int_{t-\rho(t)}^{t} K(t-s) h(y(s)) d s \\
& +2 y^{T}(t) D P D \int_{t-\sigma}^{t} y(u) d u
\end{aligned}
$$

$$
\begin{aligned}
& -2\left[\int_{t-\sigma}^{t} y(u) d u\right]^{T} \operatorname{DPAf}(y(t)) \\
& -2\left[\int_{t-\sigma}^{t} y(u) d u\right]^{T} \operatorname{DPBg}(y(t-\tau(t))) \\
& -2\left[\int_{t-\sigma}^{t} y(u) d u\right]^{T} \operatorname{DPW} \int_{t-\rho(t)}^{t} K(t-s) h(y(s)) d s .
\end{aligned}
$$

It follows from Lemma 3 that

$$
\begin{aligned}
& D^{+} V_{2} \\
& =\sigma^{2} y^{T}(t) Q_{2} y(t) \\
& \quad-\sigma \int_{t-\sigma}^{t} y^{T}(u) Q_{2} y(u) d u+y^{T}(t) Q_{3} y(t) \\
& \quad-y^{T}(t-\sigma) Q_{3} y(t-\sigma) \\
& \leq \sigma^{2} y^{T}(t) Q_{2} y(t)-\left[\int_{t-\sigma}^{t} y(u) d u\right]^{T} Q_{2}\left[\int_{t-\sigma}^{t} y(u) d u\right] \\
& \quad+y^{T}(t) Q_{3} y(t)-y^{T}(t-\sigma) Q_{3} y(t-\sigma),
\end{aligned}
$$

$D^{+} V_{3}$

$$
\begin{aligned}
= & \int_{t-\tau(t)}^{t}\left[\begin{array}{c}
y(t-\tau(t)) \\
\gamma \dot{y}(s)
\end{array}\right]^{T}\left[\begin{array}{cc}
T_{11} & T_{12} \\
\star & T_{22}
\end{array}\right]\left[\begin{array}{c}
y(t-\tau(t)) \\
\gamma \dot{y}(s)
\end{array}\right] d s \\
= & \tau(t) y^{T}(t-\tau(t)) T_{11} y(t-\tau(t)) \\
& +2 \gamma y^{T}(t) T_{12}^{T} y(t-\tau(t)) \\
& -2 \gamma y^{T}(t-\tau(t)) T_{12}^{T} y(t-\tau(t)) \\
& +\gamma^{2} \int_{t-\tau(t)}^{t} \dot{y}^{T}(s) T_{22} \dot{y}(s) d s \\
\leq & y^{T}(t-\tau(t))\left[\tau T_{11}-2 \gamma T_{12}^{T}\right] y(t-\tau(t)) \\
& +2 \gamma y^{T}(t) T_{12}^{T} y(t-\tau(t)) \\
& +\gamma^{2} \int_{t-\tau}^{t} \dot{y}^{T}(s) T_{22} \dot{y}(s) d s,
\end{aligned}
$$

$D^{+} V_{4}$

$$
\begin{aligned}
& =\gamma^{2} \tau \dot{y}^{T}(t) T_{22} \dot{y}(t)-\gamma^{2} \int_{-\tau}^{0} \dot{y}^{T}(t+u) T_{22} \dot{y}(t+u) d u \\
& =\gamma^{2} \tau \dot{y}^{T}(t) T_{22} \dot{y}(t)-\gamma^{2} \int_{t-\tau}^{t} \dot{y}^{T}(s) T_{22} \dot{y}(s) d s
\end{aligned}
$$

$D^{+} V_{5}$

$=\rho^{2} h^{T}(y(t)) K(0) Z K(0) h(y(t))$ 


$$
\begin{aligned}
& -\rho \int_{t-\rho}^{t} h^{T}(y(s)) K(t-s) Z K(t-s) h(y(s)) d s \\
= & \rho^{2} h^{T}(y(t)) K(0) Z K(0) h(y(t)) \\
- & \rho\left[\int_{t-\rho}^{t-\rho(t)} h^{T}(y(s)) K(t-s) Z K(t-s) h(y(s)) d s\right. \\
& \left.\quad+\int_{t-\rho(t)}^{t} h^{T}(y(s)) K(t-s) Z K(t-s) h(y(s)) d s\right] \\
\leq & \rho^{2} h^{T}(y(t)) K(0) Z K(0) h(y(t)) \\
- & \frac{\rho}{\rho-\rho(t)}\left(\int_{t-\rho}^{t-\rho(t)} K(t-s) h(y(s)) d s\right)^{T} \\
& \times Z\left(\int_{t-\rho}^{t-\rho(t)} K(t-s) h(y(s)) d s\right) \\
- & \frac{\rho}{\rho(t)}\left(\int_{t-\rho(t)}^{t} K(t-s) h(y(s)) d s\right)^{T} \\
& \times Z\left(\int_{t-\rho(t)}^{t} K(t-s) h(y(s)) d s\right) .
\end{aligned}
$$

In addition, we note that

$$
\begin{aligned}
0= & 2[y(t)+\gamma \dot{y}(t)]^{T} Q_{1}[-\dot{y}(t)+\dot{y}(t)] \\
= & 2[y(t)+\gamma \dot{y}(t)]^{T} Q_{1} \\
& \times[-\dot{y}(t)-D y(t-\sigma)+A f(y(t)) \\
& +B g(y(t-\tau(t))) \\
& \left.\quad+W \int_{t-\rho(t)}^{t} K(t-s) h(y(s)) d s\right] \\
= & -2 y^{T}(t) Q_{1} \dot{y}(t)-2 y^{T}(t) Q_{1} C y(t-\sigma) \\
& +2 y^{T}(t) Q_{1} A f(y(t))+2 y^{T}(t) Q_{1} B g(y(t-\tau(t))) \\
& +2 y^{T}(t) Q_{1} W \int_{t-\rho(t)}^{t} K(t-s) h(y(s)) d s \\
& -2 \gamma \dot{y}^{T}(t) Q_{1} \dot{y}(t) \\
& -2 \gamma \dot{y}^{T}(t) Q_{1} C y(t-\sigma)+2 \gamma \dot{y}^{T}(t) Q_{1} A f(y(t)) \\
& +2 \gamma \dot{y}^{T}(t) Q_{1} B g(y(t-\tau(t))) \\
& +2 \gamma \dot{y}^{T}(t) Q_{1} W \int_{t-\rho(t)}^{t} K(t-s) h(y(s)) d s .
\end{aligned}
$$

For simplicity, we denote

$$
\begin{aligned}
& \xi_{1}(t)=\int_{t-\rho(t)}^{t} K(t-s) h(y(s)) d s, \\
& \xi_{2}(t)=\int_{t-\rho}^{t-\rho(t)} K(t-s) h(y(s)) d s .
\end{aligned}
$$

By applying Lemma 4 , for any $t>0$ with $0 \leq \tau(t) \leq \tau, 0 \leq$ $\rho(t) \leq \rho$, we have

$$
\begin{aligned}
& -\frac{\rho}{\rho-\rho(t)} \xi_{2}(t)^{T} Z \xi_{2}(t)-\frac{\rho}{\rho(t)} \xi_{1}(t)^{T} Z \xi_{1}(t) \\
& \leq 2 \xi^{T}(t) \chi_{1} \xi_{2}(t)+\frac{\rho-\rho(t)}{\rho} \xi^{T}(t) \chi_{1} Z^{-1} \chi_{1}^{T} \xi(t) \\
& \quad+\frac{\rho(t)}{\rho} \xi^{T}(t) \chi_{2} Z^{-1} \chi_{2}^{T} \xi(t) \\
& \quad+2 \xi^{T}(t) \chi_{2} \xi_{1}(t),
\end{aligned}
$$

where

$$
\begin{gathered}
\xi(t)=\left(y(t), \dot{y}(t), y(t-\tau(t)), y(t-\sigma), \xi_{2}(t),\right. \\
\quad \int_{t-\sigma}^{t} y(s) d s, f(y(t)), g(y(t-\tau(t))), \\
\left.h(g(t)), \xi_{1}(t) d s\right)^{T} .
\end{gathered}
$$

Moreover, for any $n \times n$ diagonal matrices, $U_{1}>0, U_{2}>0$, and $U_{3}>0$, the following inequality holds by the methods in [40]:

$$
\begin{aligned}
& \left\{\left[\begin{array}{c}
y(t) \\
f(y(t))
\end{array}\right]^{T}\left[\begin{array}{cc}
-U_{1} \Sigma_{1} & U_{1} \Sigma_{2} \\
\star & -U_{1}
\end{array}\right]\left[\begin{array}{c}
y(t) \\
f(y(t))
\end{array}\right]\right. \\
& +\left[\begin{array}{c}
y(t-\tau(t)) \\
g(y(t-\tau(t)))
\end{array}\right]^{T}\left[\begin{array}{cc}
-U_{2} \Sigma_{3} & U_{2} \Sigma_{4} \\
\star & -U_{2}
\end{array}\right] \\
& \cdot\left[\begin{array}{c}
y(t-\tau(t)) \\
g(y(t-\tau(t)))
\end{array}\right]+\left[\begin{array}{c}
y(t) \\
h(y(t))
\end{array}\right]^{T} \\
& \left.\times\left[\begin{array}{cc}
-U_{3} \Sigma_{5} & U_{3} \Sigma_{6} \\
\star & -U_{3}
\end{array}\right]\left[\begin{array}{c}
y(t) \\
h(y(t))
\end{array}\right]\right\} \geq 0 .
\end{aligned}
$$

Combining (45)-(53), one may deduce that

$$
D^{+} V \leq \xi^{T}(t) \Xi(t) \xi(t), \quad t \in\left[t_{k-1}, t_{k}\right), k \in \mathbb{Z}_{+},
$$




$$
\begin{aligned}
& \Xi(t)= \Xi \frac{\rho-\rho(t)}{\rho} \chi_{1} Z^{-1} \chi_{1}^{T}+\frac{\rho(t)}{\rho} \chi_{2} Z^{-1} \chi_{2}^{T}, \\
& \Xi=\left[\begin{array}{cccccccccc}
\Pi_{11} & -Q_{1} & \gamma T_{12}^{T} & -Q_{1} D & X_{1} & D P D & \Pi_{17} & \Pi_{18} & U_{3} \Sigma_{6} & \Pi_{1,10} \\
\star & \Pi_{22} & 0 & -\gamma Q_{1} D & X_{2} & 0 & \gamma Q_{1} A & \gamma Q_{1} B & 0 & \Pi_{2,10} \\
\star & \star & \Pi_{33} & 0 & 0 & 0 & 0 & U_{2} \Sigma_{4} & 0 & 0 \\
\star & \star & \star & -Q_{3} & 0 & 0 & 0 & 0 & 0 & 0 \\
\star & \star & \star & \star & \Pi_{55} & 0 & 0 & 0 & 0 & 0 \\
\star & \star & \star & \star & \star & -Q_{2} & -D P A & -D P B & 0 & -D P W \\
\star & \star & \star & \star & \star & \star & -U_{1} & 0 & 0 & 0 \\
\star & \star & \star & \star & \star & \star & \star & -U_{2} & 0 & 0 \\
\star & \star & \star & \star & \star & \star & \star & \star & \Pi_{99} & 0 \\
\star & \star & \star & \star & \star & \star & \star & \star & \star & \Pi_{10,10}
\end{array}\right] \\
& \\
& \xi(t)= \\
&\left.y(t), \dot{y}(t), y(t-\tau(t)), y(t-\sigma), \xi_{2}(t), \int_{t-\sigma}^{t} y(s) d s, f(y(t)), g(y(t-\tau(t))), h(g(t)), \xi_{1}(t) d s\right)^{T} .
\end{aligned}
$$

From Lemma 5 we obtain that $\Xi(t)<0$ if the following inequalities hold simultaneously:

$$
\Xi+\chi_{1} Z^{-1} \chi_{1}^{T}<0, \quad \Xi+\chi_{2} Z^{-1} \chi_{2}^{T}<0
$$

Based on the well-known Schur complements [36], we get the fact that (56) is equivalent to (42). Therefore, $\Xi(t)<0, t \in$ $\left[t_{k-1}, t_{k}\right), k \in \mathbb{Z}_{+}$.

For arbitrary $t>0$, without loss of generality, we set $t \in$ $\left[t_{n-1}, t_{n}\right)$, for some $n \in \mathbb{Z}_{+}$. Then integrating inequality (54) at each interval $\left[t_{k-1}, t_{k}\right), 1 \leq k \leq n-1$, and $\left[t_{n-1}, t\right)$, we derive

$$
\begin{gathered}
V\left(t_{1}^{-}\right) \leq V(0)+\int_{0}^{t_{1}} \xi^{T}(u) \Xi(t) \xi(u) d u, \\
V\left(t_{2}^{-}\right) \leq V\left(t_{1}\right)+\int_{t_{1}}^{t_{2}} \xi^{T}(u) \Xi(t) \xi(u) d u, \\
\vdots \\
V\left(t_{n-1}^{-}\right) \leq V\left(t_{n-2}\right)+\int_{t_{n-2}}^{t_{n-1}} \xi^{T}(u) \Xi(t) \xi(u) d u, \\
V(t) \leq V\left(t_{n-1}\right)+\int_{t_{n-1}}^{t} \xi^{T}(u) \Xi(t) \xi(u) d u,
\end{gathered}
$$

which implies that

$$
\begin{aligned}
V(t) \leq & V(0)+\int_{0}^{t} \xi^{T}(u) \Xi(t) \xi(u) d u \\
& +\sum_{0<t_{k} \leq t}\left[V\left(t_{k}\right)-V\left(t_{k}^{-}\right)\right], \quad t \geq 0 .
\end{aligned}
$$

Now, in order to analyze (58), we consider the change of $V$ at impulse times $t_{k}, k \in \mathbb{Z}_{+}$.
Firstly, it follows from (41) that

$$
\begin{aligned}
& {\left[\begin{array}{cc}
P & \left(I-J_{k}\right) P \\
\star & P
\end{array}\right] \geq 0} \\
& \quad \Longleftrightarrow\left[\begin{array}{cc}
I & -\left(I-J_{k}\right)^{T} \\
0 & I
\end{array}\right]\left[\begin{array}{cc}
P & \left(I-J_{k}\right) P \\
\star & P
\end{array}\right]\left[\begin{array}{cc}
I & 0 \\
-\left(I-J_{k}\right) & I
\end{array}\right] \geq 0 \\
& \\
& \Longleftrightarrow\left[\begin{array}{cc}
P-\left(I-J_{k}\right)^{T} P\left(I-J_{k}\right) & 0 \\
\star & P
\end{array}\right] \geq 0 \\
& \quad \Longleftrightarrow P-\left(I-J_{k}\right)^{T} P\left(I-J_{k}\right)>0 .
\end{aligned}
$$

Secondly, from model (39), it can be obtained that

$$
\begin{aligned}
y\left(t_{k}\right)- & D \int_{t_{k}-\sigma}^{t_{k}} y(u) d u \\
= & y\left(t_{k}^{-}\right)-J_{k}\left[y\left(t_{k}^{-}\right)-D \int_{t_{k}-\sigma}^{t_{k}} y(u) d u\right] \\
& -D \int_{t_{k}-\sigma}^{t_{k}} y(u) d u \\
= & \left(I-J_{k}\right)\left[y\left(t_{k}^{-}\right)-D \int_{t_{k}-\sigma}^{t_{k}} y(u) d u\right],
\end{aligned}
$$

which together with (59) yields

$$
\begin{aligned}
V_{1}\left(t_{k}\right)= & {\left[y\left(t_{k}\right)-D \int_{t_{k}-\sigma}^{t_{k}} y(u) d u\right]^{T} } \\
& \times P\left[y\left(t_{k}\right)-D \int_{t_{k}-\sigma}^{t_{k}} y(u) d u\right] \\
= & {\left[y\left(t_{k}^{-}\right)-D \int_{t_{k}-\sigma}^{t_{k}} y(u) d u\right]^{T}\left(I-J_{k}\right)^{T} P\left(I-J_{k}\right) } \\
& \times\left[y\left(t_{k}^{-}\right)-D \int_{t_{k}-\sigma}^{t_{k}} y(u) d u\right]
\end{aligned}
$$




$$
\begin{aligned}
\leq & {\left[y\left(t_{k}^{-}\right)-D \int_{t_{k}-\sigma}^{t_{k}} y(u) d u\right]^{T} } \\
& \times P\left[y\left(t_{k}^{-}\right)-D \int_{t_{k}-\sigma}^{t_{k}} y(u) d u\right] \\
= & V_{1}\left(t_{k}^{-}\right) .
\end{aligned}
$$

Thus, we can deduce that

$$
V\left(t_{k}\right) \leq V\left(t_{k}^{-}\right), \quad k \in \mathbb{Z}_{+} .
$$

Substituting the above inequality to (58), it yields

$$
V(t)-\int_{0}^{t} \xi^{T}(u) \Xi(t) \xi(u) d u \leq V(0), \quad t \geq 0 .
$$

Applying Lemma 3 and (63), we have

$$
\begin{aligned}
\left\|D \int_{t-\sigma}^{t} y(u) d u\right\|^{2} \\
\quad=\left[D \int_{t-\sigma}^{t} y(u) d u\right]^{T}\left[D \int_{t-\sigma}^{t} y(u) d u\right] \\
\leq \lambda_{\max }\left(D^{2}\right)\left[\int_{t-\sigma}^{t} y(s) d s\right]^{T}\left[\int_{t-\sigma}^{t} y(s) d s\right] \\
\leq \frac{\lambda_{\max }\left(D^{2}\right)}{\lambda_{\min }\left(Q_{3}\right)}\left[\int_{t-\sigma}^{t} y(s) d s\right]^{T} Q_{3}\left[\int_{t-\sigma}^{t} y(s) d s\right] \\
\leq \sigma \frac{\lambda_{\max }\left(D^{2}\right)}{\lambda_{\min }\left(Q_{3}\right)} \int_{t-\sigma}^{t} y(s) Q_{3} y(s) d s \\
\leq \sigma \frac{\lambda_{\max }\left(D^{2}\right)}{\lambda_{\min }\left(Q_{3}\right)} V_{2}(t) \leq \sigma \frac{\lambda_{\max }\left(D^{2}\right)}{\lambda_{\min }\left(Q_{3}\right)} V(t) \\
\leq \sigma \frac{\lambda_{\max }\left(D^{2}\right)}{\lambda_{\min }\left(Q_{3}\right)} V(0), \quad t \geq 0 .
\end{aligned}
$$

Similarly,

$$
\begin{aligned}
\| y(u) & -D \int_{t-\sigma}^{t} y(u) d u \|^{2} \\
& =\left[y(u)-D \int_{t-\sigma}^{t} y(u) d u\right]^{T}\left[y(u)-D \int_{t-\sigma}^{t} y(u) d u\right] \\
& \leq \frac{V_{1}(t)}{\lambda_{\text {min }}(P)} \leq \frac{V(t)}{\lambda_{\text {min }}(P)} \leq \frac{V(0)}{\lambda_{\min }(P)}, \quad t \geq 0 .
\end{aligned}
$$

Hence, it can be obtained that

$$
\begin{aligned}
\|y(t)\| & \leq\left\|D \int_{t-\sigma}^{t} y(u) d u\right\|+\left\|y(u)-D \int_{t-\sigma}^{t} y(u) d u\right\| \\
& \leq \sqrt{\frac{\lambda_{\max }\left(D^{2}\right)}{\lambda_{\min }\left(Q_{3}\right)} V(0)+\sqrt{\frac{V(0)}{\lambda_{\min }(P)}}}<\infty, \quad t \geq 0,
\end{aligned}
$$

where

$$
\begin{aligned}
V(0)= & {\left[y(0)-D \int_{-\sigma}^{0} y(u) d u\right]^{T} P\left[y(0)-D \int_{-\sigma}^{0} y(u) d u\right] } \\
& +\sigma \int_{-\sigma}^{0} \int_{s}^{0} y^{T}(u) Q_{2} y(u) d u d s \\
& +\int_{-\sigma}^{0} y^{T}(s) Q_{3} y(s) d s \\
& +\gamma^{2} \int_{-\tau}^{0} \int_{u}^{0} \dot{y}^{T}(s) T_{22} \dot{y}(s) d s d u \\
& +\rho \int_{-\rho}^{0} \int_{u}^{0} h^{T}(y(s)) K(-s) Z K(-s) h(y(s)) d s d u \\
\leq & \left\{2 \lambda_{\max }(P)\left(1+\sigma^{2} \max _{i \in \Lambda} d_{i}\right)+\frac{1}{2} \sigma^{3} \lambda_{\max }\left(Q_{2}\right)\right. \\
& +\sigma \lambda_{\max }\left(Q_{3}\right)+\frac{1}{2} \gamma^{2} \tau^{2} \lambda_{\max }\left(T_{22}\right) \\
& \left.+\frac{1}{2} \rho^{3} \lambda_{\max }(Z)\right\}\|\varphi\|_{\eta}^{2}<\infty .
\end{aligned}
$$

So the solution $y(t)$ of models (1) and (4) is uniformly bounded on $[0, \infty)$. Thus, considering the continuity of activation function $f$ (i.e., $\left(H_{3}\right)$ ), it can be deduced from system (38) that there exists some constant $M>0$ such that $\|\dot{y}(t)\| \leq M, t \in\left[t_{k-1}, t_{k}\right), k \in \mathbb{Z}_{+}$. It implies that, $\left|\dot{y}_{i}(t)\right| \leq M$, $t \in\left[t_{k-1}, t_{k}\right), k \in \mathbb{Z}_{+}$, and $i \in \Lambda$, where $\dot{y}$ denotes the right hand derivative of $y$ at impulsive times.

Finally, we can prove that $\|y(t)\| \rightarrow 0$ as $t \rightarrow \infty$, which is similar to the corresponding proof in the literature [25]. Here we omit it. Therefore, the zero solution of (38) or (39) is globally asymptotically stable, which implies that models (1) and (4) have a unique equilibrium point which is globally asymptotically stable. This completes the proof.

When there is no leakage delay, that is, $\sigma=0$, models (1) and (4) become

$$
\begin{aligned}
\dot{x}(t) & =-D x(t)+A f(x(t))+B g(x(t-\tau(t))) \\
& +W \int_{t-\rho(t)}^{t} K(t-s) h(x(s)) d s+I, \\
& t>0, \quad t \neq t_{k}, \Delta x\left(t_{k}\right) \\
= & x\left(t_{k}\right)-x\left(t_{k}^{-}\right) \\
= & -I_{k}\left(x\left(t_{k}^{-}\right)-x^{*}\right), \quad k \in \mathbb{Z}_{+}, x(s) \\
= & \varphi(s), \quad s \in[-\eta, 0] .
\end{aligned}
$$

For model (68), we have the following result by Theorem 8 .

Corollary 9. Under the conditions in Theorem 7, model (68) has a unique equilibrium point which is globally asymptotically stable if there exist a constant $\gamma>0$, an $n \times n$ inverse matrix 
$Q_{1}$, four $n \times n$ matrices $P>0, Z>0, T_{11}>0$, and $T_{22}>0$, three $n \times n$ diagonal matrices $U_{1}>0, U_{2}>0$, and $U_{3}>0$, and real matrices $T_{12}, X_{l}(l=1, \ldots, 6)$ with appropriate dimension such that

$$
\begin{gathered}
{\left[\begin{array}{cc}
T_{11} & T_{12} \\
\star & T_{22}
\end{array}\right]>0,} \\
{\left[\begin{array}{cc}
P & \left(I-J_{k}\right)^{T} P \\
\star & P
\end{array}\right] \geq 0, \quad k \in \mathbb{Z}_{+},} \\
{\left[\begin{array}{cc}
\Xi & \chi_{j} \\
\star & -Z
\end{array}\right]<0, \quad j=1,2,}
\end{gathered}
$$

where

$$
\begin{gathered}
\Xi=\left[\begin{array}{cccccccc}
\Pi_{11} & -Q_{1} & \gamma T_{12}^{T} & X_{1} & \Pi_{15} & \Pi_{16} & U_{3} \Sigma_{6} & \Pi_{18} \\
\star & \Pi_{22} & 0 & X_{2} & \gamma Q_{1} A & \gamma Q_{1} B & 0 & \Pi_{28} \\
\star & \star & \Pi_{33} & 0 & 0 & U_{2} \Sigma_{4} & 0 & 0 \\
\star & \star & \star & \Pi_{44} & 0 & 0 & 0 & 0 \\
\star & \star & \star & \star & -U_{1} & 0 & 0 & 0 \\
\star & \star & \star & \star & \star & -U_{2} & 0 & 0 \\
\star & \star & \star & \star & \star & \star & \Pi_{77} & 0 \\
\star & \star & \star & \star & \star & \star & \star & \Pi_{88}
\end{array}\right], \\
\chi_{1}=\operatorname{col}\left\{X_{1}, X_{2}, O, X_{3}, O, O, O, O\right\}, \\
\chi_{2}=\operatorname{col}\left\{X_{4}, X_{5}, O, O, O, O, O, X_{6}\right\},
\end{gathered}
$$

with

$$
\begin{aligned}
& \Pi_{11}=-P D-D P-U_{1} \Sigma_{1}-U_{3} \Sigma_{5}, \\
& \Pi_{15}=P A+Q_{1} A+U_{1} \Sigma_{2}, \\
& \Pi_{16}=P B+Q_{1} B, \\
& \Pi_{18}=P W+Q_{1} W+X_{4}, \\
& \Pi_{22}=-\gamma Q_{1}-\gamma Q_{1}^{T}+\gamma^{2} \tau T_{22}, \\
& \Pi_{28}=\gamma Q_{1} W+X_{5}, \\
& \Pi_{33}=\tau T_{11}-\gamma T_{12}^{T}-\gamma T_{12}-U_{2} \Sigma_{3}, \\
& \Pi_{44}=X_{3}+X_{3}^{T}, \\
& \Pi_{77}=\rho^{2} K(0) Z K(0)-U_{3}, \\
& \Pi_{88}=X_{6}+X_{6}^{T} .
\end{aligned}
$$

Remark 10. In [25], Li et al. have investigated a class of recurrent neural networks with discrete time-varying delay and constant delay in the leakage term under impulsive perturbations. However, the bounded time-varying distributed delays were not taken into account in network models. In fact, neural networks usually have a spatial extent due to the presence of a multitude of parallel pathways with a variety of axon sizes and lengths, and hence there is a distribution of propagation delays over a period of time. Thus, the timevarying distributed delays should be taken into account to reflect more realistic dynamics. The models studied in the present paper have wider range of applications. In the future, we will further investigate the relevant models with such mixed time delays by other current approaches, such as constructing Lyapunov-Krasovskii functional with triple integral terms and introducing free-weighting matrices.

Remark 11. In [27], Wang et al. have dealt with the global asymptotic stability of neural networks with mixed time delays under impulsive perturbations. But there is a derivative restriction of transmission delay $\dot{\tau}(t) \leq c<1$, which is a conservative condition. In our results, based on a superior Lyapunov-Krasovskii functional, the criteria do not require the differentiability even differential boundedness of timevarying transmission delay $\tau(t)$. In addition, we have considered the basic properties, such as existence and uniqueness of solutions and equilibrium. So our results are less conservative and may be applied effectively in a wider range.

Remark 12. Recently, more researchers have begun to take into account the effect of time-varying leakage delay, which has essential difference from constant leakage delay, on dynamics of models. We would like to think that it may lead to more technical difficulties. How to improve the dynamics, especially the stability properties of neural networks with (time-varying) leakage delay, may be an interesting problem and requires further research.

\section{Illustrative Example}

In this section, an example is given to demonstrate the effectiveness of our results.

Example 1. Consider the following recurrent neural networks model:

$$
\begin{gathered}
\dot{x}(t)=-D x(t-\sigma)+A f(x(t))+B g(x(t-\tau(t))) \\
+W \int_{t-\rho(t)}^{t} K(t-s) h(x(s)) d s+I, \\
t>0, \quad t \neq t_{k}, \\
\Delta x\left(t_{k}\right)=x\left(t_{k}\right)-x\left(t_{k}^{-}\right)=I_{k}\left(x\left(t_{k}^{-}\right), x_{t_{k}^{-}}\right), \quad k \in \mathbb{Z}_{+},
\end{gathered}
$$

where $f=g=h=|s|, \sigma=0.08, \tau(t)=0.09-0.01[\sin t]^{*}$, $\rho(t)=0.08-0.02[\sin t]^{*}, K(s)=e^{-s}, I=(0,0)^{T}, t_{k}=0.1 k$, and $I_{k}=\operatorname{diag}(0.5,0.5), k \in \mathbb{Z}_{+}$, and parameter matrices $D, A, B$, and $W$ are given as follows:

$$
\begin{gathered}
D=\left[\begin{array}{ll}
9 & 0 \\
0 & 8
\end{array}\right], \quad A=\left[\begin{array}{cc}
1.5 & -0.3 \\
-0.1 & 0.2
\end{array}\right], \\
B=\left[\begin{array}{cc}
-0.6 & 0.4 \\
1.7 & -1.8
\end{array}\right], \quad W=\left[\begin{array}{cc}
0.2 & 0.3 \\
-0.5 & 0.15
\end{array}\right] .
\end{gathered}
$$

In this case, we know that $\sigma_{j}^{-}=\delta_{j}^{-}=\zeta_{j}^{-}=-1, \sigma_{j}^{+}=\delta_{j}^{+}=$ $\zeta_{j}^{+}=1, \sigma=0.08, \tau=0.1$, and $\rho=0.1$. Let $\gamma=2.6$; via Matlab 
LMI toolbox, the feasible solution for the LMIs in Theorem 8 is derived as follows:

$$
\begin{aligned}
& P=\left[\begin{array}{cc}
0.0017 & 0 \\
0 & 0.0026
\end{array}\right], \quad Q_{1}=\left[\begin{array}{ll}
0.0030 & 0.0001 \\
0.0001 & 0.0037
\end{array}\right], \\
& Q_{2}=\left[\begin{array}{ll}
0.8011 & 0.0002 \\
0.0002 & 0.8061
\end{array}\right], \quad Q_{3}=\left[\begin{array}{ll}
0.5982 & 0.0009 \\
0.0009 & 0.6026
\end{array}\right], \\
& Z=\left[\begin{array}{cc}
0.8887 & 0 \\
0 & 0.8887
\end{array}\right], \quad U_{1}=\left[\begin{array}{cc}
1.0046 & 0 \\
0 & 1.0046
\end{array}\right] \text {, } \\
& U_{2}=\left[\begin{array}{cc}
0.8793 & 0 \\
0 & 0.8793
\end{array}\right], \quad U_{3}=\left[\begin{array}{cc}
1.0117 & 0 \\
0 & 1.0117
\end{array}\right] \text {, } \\
& T_{11}=\left[\begin{array}{cc}
1.3262 & -0.0001 \\
-0.0001 & 1.3260
\end{array}\right], \quad T_{12}=\left[\begin{array}{cc}
0.0388 & 0 \\
0 & 0.0387
\end{array}\right] \text {, } \\
& T_{22}=\left[\begin{array}{ll}
0.0096 & 0.0002 \\
0.0002 & 0.0113
\end{array}\right] \text {, } \\
& X_{1}=10^{-3} \times\left[\begin{array}{cc}
0.0534 & 0.0807 \\
-0.1311 & 0.0400
\end{array}\right] \text {, } \\
& X_{2}=10^{-3} \times\left[\begin{array}{cc}
0.1117 & 0.1773 \\
-0.3554 & 0.1106
\end{array}\right] \text {, } \\
& X_{3}=\left[\begin{array}{cc}
-0.3176 & 0 \\
0 & -0.3176
\end{array}\right], \quad X_{4}=\left[\begin{array}{cc}
-0.0004 & -0.0007 \\
0.0012 & -0.0004
\end{array}\right] \text {, } \\
& X_{5}=\left[\begin{array}{cc}
-0.0014 & -0.0022 \\
0.0044 & -0.0014
\end{array}\right] \text {, } \\
& X_{6}=\left[\begin{array}{cc}
-0.3177 & 0 \\
0 & -0.3177
\end{array}\right] \text {. }
\end{aligned}
$$

Hence, from Theorem 8 , the unique equilibrium point $x^{*}=$ $(0,0)^{T}$ of system $(72)$ is globally asymptotically stable.

\section{Conclusion}

In this paper, we have investigated a class of impulsive neural networks with mixed time delays and generalized activation functions. Firstly, by using the contraction mapping theorem, we have given a sufficient condition to guarantee the global existence and uniqueness of the solution for the addressed neural networks. Then, a delay-independent sufficient condition for existence of the equilibrium point and some delay-dependent sufficient conditions for stability have been derived, respectively, by using topological degree theory and suitable Lyapunov-Krasovskii functional. The obtained results require neither the boundedness, monotonicity, and differentiability of the activation functions nor the differentiability of time-varying delay. Finally, an example has been given to show the effectiveness and less conservativeness of the obtained results. In the future, we will do some further research on impulsive neural network models with leakage time-varying delay and continuously distributed delay.

\section{Conflict of Interests}

The authors declare that there is no conflict of interests regarding the publication of this paper.

\section{Acknowledgment}

This work was partially supported by the National Natural Science Foundation of China Grant no. 11301304.

\section{References}

[1] K. Gopalsamy, Stability and Oscillations in Delay Differential Equations of Population Dynamics, Kluwer Academic, Dodrecht, The Netherlands, 1992.

[2] J. K. Hale and S. M. V. Lunel, Introduction to Functional Differential Equations, Springer, New York, NY, USA, 1993.

[3] S. Haykin, Neural Networks, Prentice-Hall, Englewood Cliffs, NJ, USA, 1994.

[4] V. Kolmanovskii and A. Myshkis, Introduction to the Theory and Applications of Functional-Differential Equations, Kluwer Academic, Dodrecht, The Netherlands, 1999.

[5] S.-I. Niculescu, Delay Effects on Stability: A Robust Control Approach, Springer, New York, NY, USA, 2001.

[6] V. L. Kharitonov and A. P. Zhabko, "Lyapunov-Krasovskii approach to the robust stability analysis of time-delay systems," Automatica, vol. 39, no. 1, pp. 15-20, 2003.

[7] P. Baldi and A. F. Atiya, "How delays affect neural dynamics and learning," IEEE Transactions on Neural Networks, vol. 5, no. 4, pp. 612-621, 1994.

[8] C. M. Marcus and R. M. Westervelt, "Stability of analog neural networks with delay," Physical Review A, vol. 39, no. 1, pp. 347$359,1989$.

[9] M. Wu, Y. He, J.-H. She, and G.-P. Liu, "Delay-dependent criteria for robust stability of time-varying delay systems," Automatica, vol. 40, no. 8, pp. 1435-1439, 2004.

[10] K. Gu, "A further refinement of discretized Lyapunov functional method for the stability of time-delay systems," International Journal of Control, vol. 74, no. 10, pp. 967-976, 2001.

[11] K. Gopalsamy, "Leakage delays in BAM," Journal of Mathematical Analysis and Applications, vol. 325, no. 2, pp. 1117-1132, 2007.

[12] C. Li and T. Huang, "On the stability of nonlinear systems with leakage delay," Journal of the Franklin Institute, vol. 346, no. 4, pp. 366-377, 2009.

[13] K. Gopalsamy, "Stability of artificial neural networks with impulses," Applied Mathematics and Computation, vol. 154, no. 3, pp. 783-813, 2004.

[14] R. Rakkiyappan, P. Balasubramaniam, and J. Cao, "Global exponential stability results for neutral-type impulsive neural networks," Nonlinear Analysis: Real World Applications, vol. 11, no. 1, pp. 122-130, 2010.

[15] Z.-H. Guan and G. Chen, "On delayed impulsive Hopfield neural networks," Neural Networks, vol. 12, no. 2, pp. 273-280, 1999.

[16] X. Li and M. Bohner, "Exponential synchronization of chaotic neural networks with mixed delays and impulsive effects via output coupling with delay feedback," Mathematical and Computer Modelling, vol. 52, no. 5-6, pp. 643-653, 2010.

[17] Q. Zhou, "Global exponential stability of BAM neural networks with distributed delays and impulses," Nonlinear Analysis: Real World Applications, vol. 10, no. 1, pp. 144-153, 2009. 
[18] Z.-C. Yang and D.-Y. Xu, "Global exponential stability of Hopfield neural networks with variable delays and impulsive effects," Applied Mathematics and Mechanics, vol. 27, no. 11, pp. 1517-1522, 2006.

[19] D. Xu and Z. Yang, "Impulsive delay differential inequality and stability of neural networks," Journal of Mathematical Analysis and Applications, vol. 305, no. 1, pp. 107-120, 2005.

[20] X. Liu, K. L. Teo, and B. Xu, "Exponential stability of impulsive high-order Hopfield-type neural networks with time-varying delays," IEEE Transactions on Neural Networks, vol. 16, no. 6, pp. 1329-1339, 2005.

[21] Z. Huang and Y. Xia, "Global exponential stability of BAM neural networks with transmission delays and nonlinear impulses," Chaos, Solitons \& Fractals, vol. 38, no. 2, pp. 489-498, 2008.

[22] D. Yue, E. Tian, Y. Zhang, and C. Peng, "Delay-distributiondependent stability and stabilization of T-S fuzzy systems with probabilistic interval delay," IEEE Transactions on Systems, Man, and Cybernetics B: Cybernetics, vol. 39, no. 2, pp. 503-516, 2009.

[23] S. Peng, "Global attractive periodic solutions of BAM neural networks with continuously distributed delays in the leakage terms," Nonlinear Analysis: Real World Applications, vol. 11, no. 3, pp. 2141-2151, 2010.

[24] X. Li and J. Cao, "Delay-dependent stability of neural networks of neutral type with time delay in the leakage term," Nonlinearity, vol. 23, no. 7, pp. 1709-1726, 2010.

[25] X. Li, X. Fu, P. Balasubramaniam, and R. Rakkiyappan, "Existence, uniqueness and stability analysis of recurrent neural networks with time delay in the leakage term under impulsive perturbations," Nonlinear Analysis: Real World Applications, vol. 11, no. 5, pp. 4092-4108, 2010.

[26] X. Li, R. Rakkiyappan, and P. Balasubramaniam, "Existence and global stability analysis of equilibrium of fuzzy cellular neural networks with time delay in the leakage term under impulsive perturbations," Journal of the Franklin Institute, vol. 348, no. 2, pp. 135-155, 2011.

[27] Y. Wang, C.-D. Zheng, and E. Feng, "Stability analysis of mixed recurrent neural networks with time delay in the leakage term under impulsive perturbations," Neurocomputing, vol. 119, pp. 454-461, 2013.

[28] S. Lakshmanan, J. H. Park, H. Y. Jung, and P. Balasubramaniam, "Design of state estimator for neural networks with leakage, discrete and distributed delays," Applied Mathematics and Computation, vol. 218, no. 22, pp. 11297-11310, 2012.

[29] P. Balasubramaniam, V. Vembarasan, and R. Rakkiyappan, "Leakage delays in T-S fuzzy cellular neural networks," Neural Processing Letters, vol. 33, no. 2, pp. 111-136, 2011.

[30] P. Balasubramaniam, V. Vembarasan, and R. Rakkiyappan, "Global robust asymptotic stability analysis of uncertain switched Hopfield neural networks with time delay in the leakage term," Neural Computing \& Applications, vol. 21, no. 7, pp. 1593-1616, 2012.

[31] R. Rakkiyappan and P. Balasubramaniam, "LMI conditions for global asymptotic stability results for neutral-type neural networks with distributed time delays," Applied Mathematics and Computation, vol. 204, no. 1, pp. 317-324, 2008.

[32] X. Li, "Existence and global exponential stability of periodic solution for impulsive Cohen-Grossberg-type BAM neural networks with continuously distributed delays," Applied Mathematics and Computation, vol. 215, no. 1, pp. 292-307, 2009.

[33] V. Lakshmikantham, D. D. Bainov, and P. S. Simeonov, Theory of Impulsive Differential Equations, World Scientific, Singapore, 1989.
[34] A. O. Ignatyev, "On the stability of invariant sets of systems with impulse effect," Nonlinear Analysis: Theory, Methods and Applications, vol. 69, no. 1, pp. 53-72, 2008.

[35] X. Fu, B. Yan, and Y. Liu, Introduction of Impulsive Differential Systems, Science Press, Beijing, China, 2005.

[36] S. Boyd, L. El Ghaoui, E. Feron, and V. Balakrishnan, Linear Matrix Inequality in System and Control Theory, SIAM, Philadelphia, Pa, USA, 1994.

[37] D. Guo, Nonlinear Functional Analysis, Shangdon Science and Technology Press, Jinan, China, 1985.

[38] K. Gu, "An integral inequality in the stability problem of time-delay systems," in Proceedings of the 39th IEEE Confernce on Decision and Control, pp. 2805-2810, Sydney, Australia, December 2000.

[39] J. Shen, Y. Liu, and J. Li, "Asymptotic behavior of solutions of nonlinear neutral differential equations with impulses," Journal of Mathematical Analysis and Applications, vol. 332, no. 1, pp. 179-189, 2007.

[40] T. Li, S.-M. Fei, and Q. Zhu, "Design of exponential state estimator for neural networks with distributed delays," Nonlinear Analysis: Real World Applications, vol. 10, no. 2, pp. 1229-1242, 2009. 


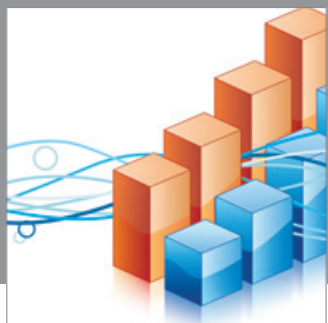

Advances in

Operations Research

mansans

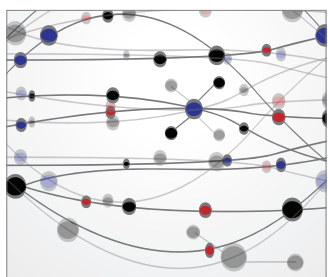

The Scientific World Journal
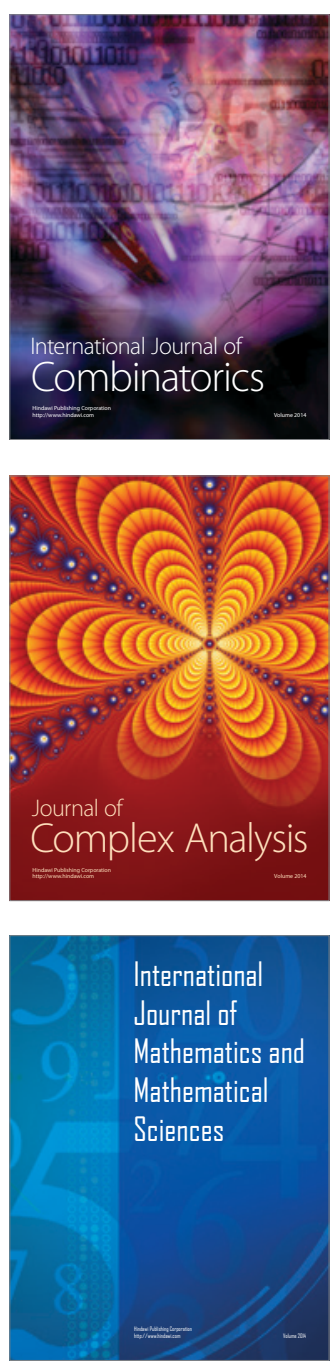
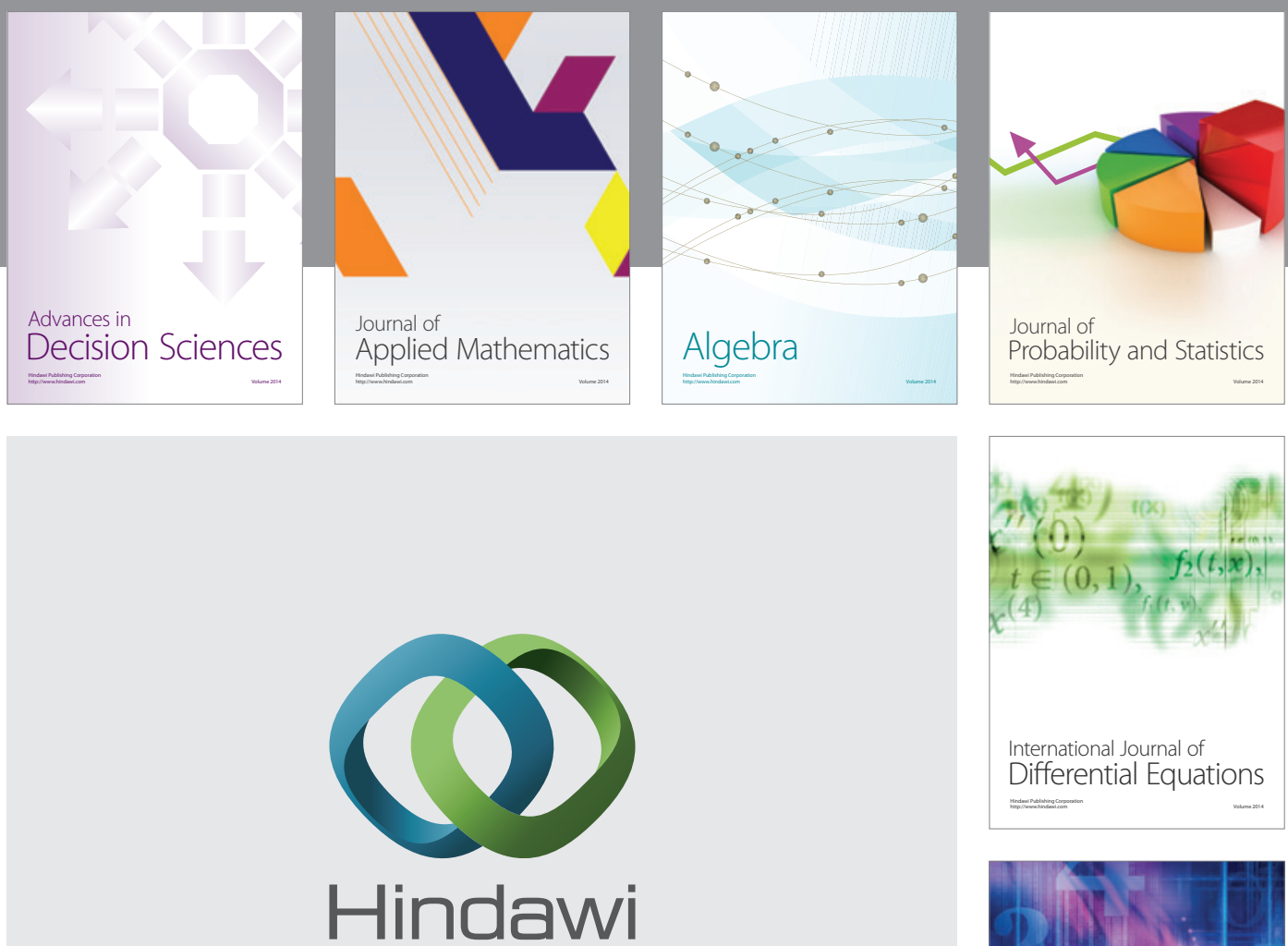

Submit your manuscripts at http://www.hindawi.com
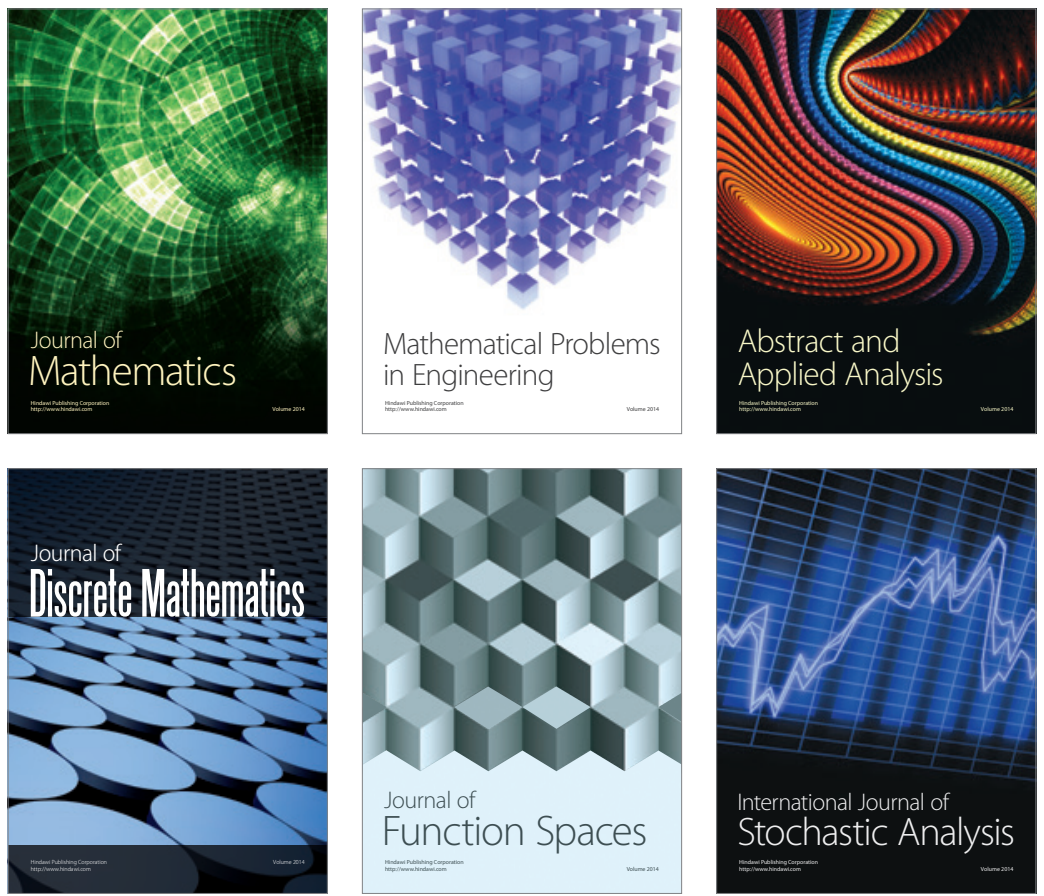

Journal of

Function Spaces

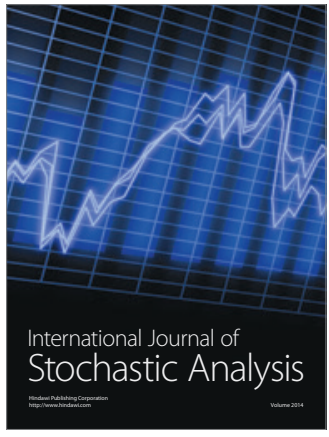

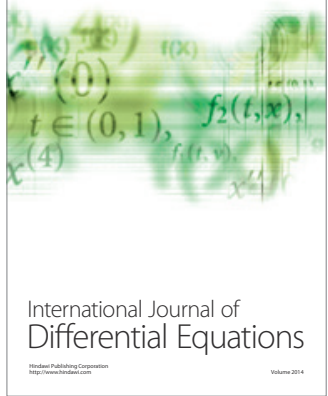
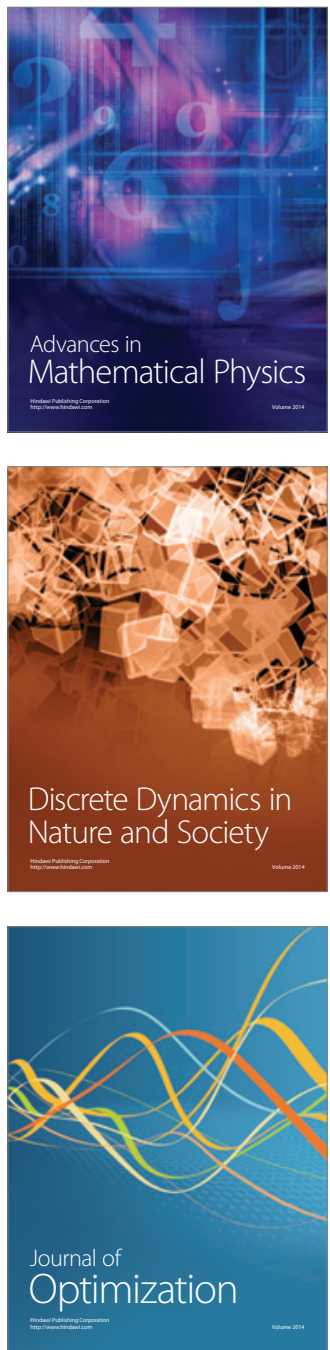